\title{
Maximal neutrino mixing from a minimal flavor symmetry
}

\author{
Alfredo Aranda* and Christopher D. Carone ${ }^{\dagger}$ \\ Nuclear and Particle Theory Group, Department of Physics, College of William and Mary, Williamsburg, Virginia 23187-8795
}

Richard F. Lebed

Jefferson Lab, 12000 Jefferson Avenue, Newport News, Virginia 23606

(Received 4 February 2000; published 12 June 2000)

\begin{abstract}
We study a number of models, based on a non-Abelian discrete group, that successfully reproduce the simple and predictive Yukawa textures usually associated with $\mathrm{U}(2)$ theories of flavor. These models allow for solutions to the solar and atmospheric neutrino problems that do not require altering successful predictions for the charged fermions or introducing sterile neutrinos. Although Yukawa matrices are hierarchical in the models we consider, the mixing between second- and third-generation neutrinos is naturally large. We first present a quantitative analysis of a minimal model proposed in earlier work, consisting of a global fit to fermion masses and mixing angles, including the most important renormalization group effects. We then propose two new variant models: The first reproduces all important features of the $S U(5) \times U(2)$ unified theory with neither SU(5) nor U(2). The second demonstrates that discrete subgroups of SU(2) can be used in constructing viable supersymmetric theories of flavor without scalar universality even though $\mathrm{SU}(2)$ by itself cannot.
\end{abstract}

PACS number(s): 11.30.Hv, 12.15.Ff, 12.60.Jv, 14.60.Pq

\section{INTRODUCTION}

It is possible that the observed hierarchy of fermion masses and mixing angles originates from the spontaneous breakdown of a new symmetry $G_{f}$ that acts horizontally across the three standard model generations. Ideally, all Yukawa couplings except that of the top quark are forbidden by $G_{f}$ invariance at high energies; the remaining ones are generated when a set of fields $\phi$ that transform nontrivially under $G_{f}$ develops vacuum expectation values (VEVs). A hierarchy in couplings is obtained if $G_{f}$ is broken sequentially at energy scales $\mu_{i}$ through a series of nested subgroups $H_{i}$, such that

$$
\stackrel{\mu_{1}}{\mu_{2} \stackrel{\mu_{3}}{G_{f} \rightarrow H_{1} \rightarrow H_{2} \rightarrow \cdots} \text { for } \mu_{1}>\mu_{2}>\mu_{3} \cdots .}
$$

At each stage of the symmetry breaking there is an associated small dimensionless parameter $\left\langle\phi_{i}\right\rangle / M_{f}$, where $\phi_{i}$ is a "flavon" field whose VEV is responsible for the breaking $H_{i-1} \rightarrow H_{i}$, and where $M_{f}$ is the ultraviolet cutoff of the $G_{f}$-invariant effective theory. The ratios $\phi_{i} / M_{f}$ appear in higher-dimension operators that contribute to Yukawa couplings in the low-energy theory. For example, the superpotential term

$$
\frac{1}{M_{f}} Q_{3} H_{D} \phi_{b} D_{3}
$$

leads to a bottom quark Yukawa coupling of order $\left\langle\phi_{b}\right\rangle / M_{f}$. The most general set of operators involving the fields of the minimal supersymmetric standard model (MSSM) and the $\phi$

\footnotetext{
*Email address: fefo@physics.wm.edu

†Email address: carone@physics.wm.edu

*Email address: lebed@jlab.org
}

fields must provide for Yukawa textures that are phenomenologically viable. If flavor universality of scalar superpartner masses is not simply a consequence of the mechanism by which supersymmetry breaking is mediated [1-4], then a successful model must also explain why these scalars do not contribute to flavor-changing neutral current processes at unacceptable levels.

Models with horizontal symmetries have been proposed with $G_{f}$ either gauged or global, continuous or discrete, Abelian or non-Abelian, or some appropriate combination thereof $[5,6]$. Abelian flavor symmetries have been used successfully to explain the absence of supersymmetric flavorchanging processes by aligning the fermion and sfermion mass matrices [5]. However, the freedom to choose a number of new U(1) charges for each MSSM matter field represents so much freedom that these models seem ad hoc, at least from a low-energy point of view. Non-Abelian symmetries are more restrictive, as the Yukawa matrices generally decompose into a smaller number of irreducible $G_{f}$ representations. Thus, it is not unreasonable to expect that minimal models exist that are both successful and aesthetically compelling. This is the primary motivation for the current work.

In non-Abelian flavor models, the existence of three generations of matter fields, the heaviness of the top quark, and the absence of supersymmetric flavor-changing processes together suggest a $\mathbf{2} \oplus \mathbf{1}$ representation structure for the MSSM matter fields. With this choice it is not only possible to distinguish the third generation, but also to achieve an exact degeneracy between superparticles of the first two generations when $G_{f}$ is unbroken. In the low-energy theory, this degeneracy is lifted by the same small symmetry-breaking parameters that determine the light fermion Yukawa couplings, so that flavor changing neutral current (FCNC) effects remain adequately suppressed, even with superparticle masses less than $1 \mathrm{TeV}$.

A particularly elegant model of this type considered in the literature assumes the continuous, global symmetry $G_{f}$ 
$=\mathrm{U}(2)[8-10]$. Quarks and leptons are assigned to $\mathbf{2} \oplus \mathbf{1}$ representations, so that in tensor notation one may represent the three generations of any matter field by $F^{a}+F^{3}$, where $a$ is a $\mathrm{U}(2)$ index, and $F$ is $Q, U, D, L$, or $E$. A set of flavons is introduced consisting of $\phi_{a}, S_{a b}$, and $A_{a b}$, where $\phi$ is a $\mathrm{U}(2)$ doublet, and $S(A)$ is a symmetric (antisymmetric) $\mathrm{U}(2)$ triplet (singlet). The doublet and triplet flavons acquire the VEVs

$$
\frac{\langle\phi\rangle}{M_{f}}=\left(\begin{array}{l}
0 \\
\epsilon
\end{array}\right) \quad \text { and } \frac{\langle S\rangle}{M_{f}}=\left(\begin{array}{ll}
0 & 0 \\
0 & \epsilon
\end{array}\right) \text {, }
$$

the most general set of nonvanishing entries consistent with an unbroken $U(1)$ symmetry that rotates all first generationfields by a phase. This residual U(1) symmetry is broken at a somewhat lower scale by the flavon $A$ :

$$
\frac{\langle A\rangle}{M_{f}}=\left(\begin{array}{cc}
0 & \epsilon^{\prime} \\
-\epsilon^{\prime} & 0
\end{array}\right),
$$

where $\epsilon^{\prime}<\epsilon$. Thus, the sequential breaking

$$
\mathrm{U}(2) \stackrel{\epsilon}{\rightarrow} \mathrm{U}(1) \stackrel{\epsilon}{\rightarrow} \text { nothing }
$$

yields a Yukawa texture for the down quarks, for example, of the form

$$
Y_{D} \approx\left(\begin{array}{ccc}
0 & d_{1} \epsilon^{\prime} & 0 \\
-d_{1} \epsilon^{\prime} & d_{2} \epsilon & d_{3} \epsilon \\
0 & d_{4} \epsilon & d_{5}
\end{array}\right) \xi
$$

where $d_{1}, \ldots, d_{5}$ are $O(1)$ coefficients. With the choice $\epsilon$ $\approx 0.02$ and $\epsilon^{\prime} \approx 0.004$, this texture achieves the correct hierarchy in down quark mass eigenvalues and gives contributions of the appropriate size to entries of the CabibboKobayashi-Maskawa (CKM) matrix. The $O(1)$ coefficients may be determined from a global fit, as in Ref. [10]. The ratio $m_{b} / m_{t}$ is assumed to be unrelated to $\mathrm{U}(2)$ symmetry breaking, and is simply put into the low-energy theory by hand. This is accomplished by choosing the free parameter $\xi$ in Eq. (1.6).

While the form of $Y_{D}$ is viable, $\mathrm{U}(2)$ symmetry by itself cannot explain the differences between the hierarchies within $Y_{D}$ and $Y_{U}$. Quark mass ratios renormalized at the grand unified scale are given approximately by [7]

$$
m_{d}:: m_{s}:: m_{b}=\lambda^{4}:: \lambda^{2}:: 1
$$

while

$$
m_{u}:: m_{c}:: m_{t}=\lambda^{8}:: \lambda^{4}:: 1,
$$

where $\lambda \approx 0.22$ is the Cabibbo angle. Clearly, an additional suppression factor $\rho$ is required in $Y_{U}$ for those elements that contribute most significantly to the up and charm quark mass eigenvalues,

$$
Y_{U} \approx\left(\begin{array}{ccc}
0 & u_{1} \epsilon^{\prime} \rho & 0 \\
-u_{1} \epsilon^{\prime} \rho & u_{2} \epsilon \rho & u_{3} \epsilon \\
0 & u_{4} \epsilon & u_{5}
\end{array}\right),
$$

where $u_{1}, \ldots, u_{5}$ are $O(1)$ coefficients. By embedding the $\mathrm{U}(2)$ model in a grand unified theory it is possible to obtain $\rho \approx \epsilon$ naturally; the model can then accommodate all the desired fermion mass hierarchies for choices of the coefficients $u_{i}$ and $d_{i}$ that are all of order 1 [10]. For example, in an $\mathrm{SU}(5)$ grand unified theory (GUT), $Y_{U}$ is associated with the coupling 10-10-5, where the 10's represent matter fields, and the $\mathbf{5}$ is the Higgs field $H$. However,

$$
\mathbf{1 0} \otimes \mathbf{1 0}=\overline{\mathbf{5}}_{s} \oplus \overline{\mathbf{4 5}}_{a} \oplus \overline{\mathbf{5 0}}_{s}
$$

where the subscripts indicate symmetry or antisymmetry under interchange of the two $\overline{\mathbf{1 0}}$ 's. If we assume that the antisymmetric flavon $A$ is an $\mathrm{SU}(5)$ singlet, the product $A H$ is a $\mathbf{5}_{a}$, and does not contribute to $Y_{U}$. Similarly, if the flavon $S$ is a 75 with a VEV in the hypercharge direction in $\mathrm{SU}(5)$ space, then the part of $S H$ that contains the Higgs doublet field transforms as a $\mathbf{4 5}_{s}$, which again does not contribute to $Y_{U}$. To obtain nonvanishing couplings of the right size in the upper $2 \times 2$ block of $Y_{U}$ one introduces a singlet flavon $\Sigma$ that transforms as an SU(5) adjoint. The VEV of $S$ implies that the breakings of both $\mathrm{U}(2)$ to $\mathrm{U}(1)$ and $\mathrm{SU}(5)$ to the standard model gauge group are associated with VEVs of order $\epsilon$. Thus, it is natural to assume $\langle\Sigma\rangle \approx \epsilon$, which provides exactly the desired value of $\rho$ in Eq. (1.9). Moreover, the SU(5) assignments for $A$ and $S$ provide for a Georgi-Jarlskog mechanism [11], so that unified U(2) models successfully account for the charged lepton mass spectrum as well.

While the textures that follow from the simple two-step breaking of a U(2) flavor symmetry are indeed minimal, the original symmetry group is not. It is natural to ask whether there are small discrete groups that work equally well as horizontal symmetries. It was shown in Ref. [12] that the charged fermion Yukawa textures usually associated with $\mathrm{U}(2)$ models may be reproduced assuming the symmetry $G_{f}=T^{\prime} \times Z_{3}$, and the breaking pattern

$$
T^{\prime} \otimes Z_{3} \stackrel{\epsilon}{\rightarrow} \underset{Z_{3}^{D}}{\epsilon^{\prime}} \rightarrow \text { nothing. }
$$

Here, $T^{\prime}$ is the double tetrahedral group, a discrete subgroup of SU(2) corresponding to the symmetry of a regular tetrahedron. The factor $Z_{3}^{D}$ is the diagonal subgroup of a $Z_{3} \subset T^{\prime}$ and the additional $Z_{3}$ factor (see Sec. IV). Since $U(2)$ is isomorphic to $\mathrm{SU}(2) \times \mathrm{U}(1)$, it is not surprising that our discrete symmetry is a product of a discrete subgroup of SU(2) and a discrete subgroup of U(1). Moreover, it was argued in Ref. [12] that this symmetry is minimal in the sense that

(i) $T^{\prime}$ is the smallest discrete subgroup of $\mathrm{SU}(2)$ (and in fact the smallest group of any kind) with 1-, 2- and 3 -dimensional representations and the multiplication rule 2 $\otimes \mathbf{2}=\mathbf{3} \oplus \mathbf{1}$. These two ingredients are necessary to reproduce the successful $\mathrm{U}(2)$ textures. 
(ii) $Z_{3}$ is the smallest discrete subgroup of $\mathrm{U}(1)$ that allows $G_{f}$ to contain a subgroup forbidding all order $O\left(\epsilon^{\prime}\right)$ entries in the Yukawa textures.

The latter statement applies to models in which $T^{\prime}$ is a discrete gauge symmetry (see Sec. II); models with a global $T^{\prime}$ symmetry do not require any additional Abelian factors, as we demonstrate in Sec. VII. The use of a discrete gauge rather than global symmetries is motivated by various arguments that the latter are violated at order 1 by quantum gravitational effects [13]. In two of the models we present, $T^{\prime}$ is an anomaly-free discrete gauge symmetry, while the additional $Z_{n}$ factor is not. As in many of the Abelian models described in the literature [5], we simply assume that the $Z_{n}$ factor may be embedded in a U(1) gauge symmetry whose anomalies are cancelled by the Green-Schwarz mechanism [14]. Thus, our models may be viewed as consistent lowenergy effective theories for flavor symmetries that are local in a complete, high-energy theory.

On a more practical level, the different representation structure of $T^{\prime}$ allows for elegant solutions to the solar and atmospheric neutrino problems that do not alter the predictive quark and charged lepton Yukawa textures, nor require the introduction of sterile neutrinos. While similar results can be obtained in some $\mathrm{SO}(10) \times \mathrm{U}(2)$ models [15], we obtain our successful solutions using a much smaller symmetry structure. ${ }^{1}$ One goal of this paper is to study these solutions at a level of quantitative detail not presented in our earlier work.

In addition, we propose two new models involving $T^{\prime}$ symmetry. The first model, based on the discrete gauge symmetry $T^{\prime} \times Z_{6}$, reproduces all important features of the $\mathrm{SU}(5) \times \mathrm{U}(2)$ model without requiring a field-theoretic grand unified theory. In other words, the suppression of $m_{u}$ and $m_{c}$ in the $\mathrm{SU}(5) \times \mathrm{U}(2)$ theory described earlier is achieved in $T^{\prime} \times Z_{6}$ without $\mathrm{SU}(5)$. In addition, the ratio $m_{b} / m_{t}$, which is not explained in $\mathrm{SU}(5) \times \mathrm{U}(2)$, is predicted in our model to be of $O(\epsilon) \approx 0.02$ for $\tan \beta \sim O(1)$, where $\tan \beta$ is the ratio of Higgs field VEVs $\left\langle H_{U}\right\rangle /\left\langle H_{D}\right\rangle$. In a second model, we consider the implications of $T^{\prime}$ as a purely global flavor symmetry. Although in this case the symmetry may not be fundamental, it could still arise as an accidental symmetry at low energies. We show that it is possible to construct a viable model based on $T^{\prime}$ alone, with no additional Abelian factors. While it is well known that supersymmetric models with a continuous $\mathrm{SU}(2)$ flavor symmetry and a $\mathbf{2} \oplus \mathbf{1}$ representation structure do not have viable Yukawa textures, our global $T^{\prime}$ model demonstrates that discrete subgroups of $\mathrm{SU}(2)$ remain viable alternatives.

Our paper is organized as follows. In the next section, we discuss the meaning of discrete gauge symmetries and the relevant anomaly-cancellation constraints in the low-energy effective theory. In Secs. III and IV, we review the group theory of $T^{\prime}$ and present the minimal model described in Ref. [12]. In Sec. V we fit predictions of the model to charged fermion and neutrino masses and mixing angles, in-

\footnotetext{
${ }^{1}$ For a similar approach, see Ref. [16].
}

cluding the most significant renormalization group effects. In Sec. VI, we present the $T^{\prime} \times Z_{6}$ model that reproduces the important features of the $\mathrm{SU}(5) \times \mathrm{U}(2)$ model with neither $\mathrm{SU}(5)$ nor U(2). In Sec. VII, we show how to construct a viable global $T^{\prime}$ model with no Abelian factors. In Sec. VIII we comment on one scenario involving sterile neutrinos, and in the final section we summarize our conclusions.

\section{WHAT IS A DISCRETE GAUGE SYMMETRY?}

Let us define a discrete gauge symmetry provisionally as a discrete remnant of a spontaneously broken continuous gauge symmetry. Below the breaking scale $\Lambda$ of the continuous symmetry, the low-energy effective Lagrangian has interactions that are invariant under the unbroken discrete group, no massless gauge fields, and derivatives that transform trivially. It would seem then that this effective theory is identical to one with a purely global discrete symmetry. In this section, we review the arguments suggesting that this is not the case. We first illustrate how gauge invariance of a theory spontaneously broken to a discrete subgroup dictates the form of all terms in the low-energy effective theory, and thus renders its discrete invariance immune to wormhole dynamics. We then show that a theory with a discrete gauge symmetry predicts topological defects not present in a theory with a global symmetry, and that these play an important role in demonstrating that discrete gauge charges leave quantummechanical hair on black holes. Both observations suggest that discrete gauge symmetries are viable as candidates for fundamental symmetries of nature. After reviewing these arguments we summarize the anomaly-cancellation constraints relevant to low-energy theories with discrete gauge symmetries. We use these constraints in constructing models throughout this paper.

Following a discussion by Banks [17], let us consider the low-energy effective theory that results from spontaneously breaking a U(1) gauge symmetry to a discrete subgroup. The full theory consists of two scalar fields $\chi$ and $\phi$ with $\mathrm{U}(1)$ charges $q$ and 1 , respectively, where $q$ is an integer. The Lagrangian is the usual one for an Abelian Higgs model:

$$
\begin{aligned}
\mathcal{L}= & -\frac{1}{4 g^{2}} F_{\mu \nu} F^{\mu \nu}+\left|\partial_{\mu} \chi-i q A_{\mu} \chi\right|^{2}+\left|\partial_{\mu} \phi-i A_{\mu} \phi\right|^{2} \\
& +V\left(\chi^{\dagger} \chi\right) .
\end{aligned}
$$

The potential $V$ is such that the $\chi$ field acquires a vacuum expectation value $\Lambda$. Let us rewrite the Lagrangian using the nonlinear field redefinition $\chi=(\Lambda+\sigma) e^{i \theta} / \sqrt{2}$. This yields

$$
\begin{aligned}
\mathcal{L}= & -\frac{1}{4 g^{2}} F_{\mu \nu} F^{\mu \nu}+\frac{1}{2} \partial_{\mu} \sigma \partial^{\mu} \sigma+\frac{1}{2}(\Lambda+\sigma)^{2}\left(\partial_{\mu} \theta-q A_{\mu}\right)^{2} \\
& +\left|\partial_{\mu} \phi-i A_{\mu} \phi\right|^{2}+V(\sigma),
\end{aligned}
$$

where $\sigma$ is the Higgs field and $\theta$ is the would-be longitudinal component of the $\mathrm{U}(1)$ gauge boson in unitary gauge. We choose to construct a low-energy effective theory in which the $\sigma$ field, which has a mass of order $\Lambda$, is integrated out. 
However, we retain the gauge field $A_{\mu}$ as well as the unphysical scalar field $\theta$. Although the gauge symmetry is spontaneously broken, the Lagrangian of the theory remains invariant under the local U(1) transformation

$$
\phi \rightarrow e^{i \alpha(x)} \phi, \quad A^{\mu} \rightarrow A^{\mu}+\partial_{\mu} \alpha(x), \quad \theta \rightarrow \theta+q \alpha(x) .
$$

The low-energy effective Lagrangian then consists of the kinetic terms

$$
\mathcal{L}=-\frac{1}{4 g^{2}} F_{\mu \nu} F^{\mu \nu}+\left|\partial_{\mu} \phi-i A_{\mu} \phi\right|^{2}+\frac{1}{2} \Lambda^{2}\left(\partial_{\mu} \theta-q A_{\mu}\right)^{2},
$$

as well as the most general set of gauge-invariant operators involving the fields $\phi, e^{i \theta}$, and covariant derivatives, with powers of $\Lambda$ included to obtain the correct mass dimensions. We can classify the interactions in the effective Lagrangian that involve $\phi$ into two types: terms that are invariant under global U(1) transformations on $\phi$ alone (with the other fields held fixed) and those that are not. A typical term of the first type is $\phi^{\dagger} \phi$; terms of the second type necessarily involve the $\mathrm{U}(1)$ gauge-invariant product

$$
e^{-i \theta} \phi^{q}
$$

or similar products with derivatives. Such terms are invariant under a $Z_{q}$ phase rotation of the field $\phi$ alone. Thus, gauge invariance of the low-energy theory implies that it must have an unbroken $Z_{q}$ symmetry. Since this is a consequence of a local symmetry, it cannot be violated by wormhole dynamics.

We now show that information on discrete gauge charges is not lost when a charged particle falls into a black hole. To do so, first note that the Abelian Higgs model has stable cosmic string solutions. In the case where $\phi=0$, the kinetic energy terms in Eq. (2.4) are minimized when

$$
A_{\mu}=\frac{1}{q} \partial_{\mu} \theta
$$

For nonsingular gauge field configurations, this is related to $A_{\mu}=0$ by a gauge transformation. However, singular solutions also exist; a cosmic string along the $x^{3}$ axis corresponds to

$$
A_{i}=\frac{1}{q} \epsilon_{i j} \frac{x^{j}}{x_{1}^{2}+x_{2}^{2}}, \quad i, j=1,2, \quad \theta=\arctan \left(x_{2} / x_{1}\right) .
$$

If one couples the gauge field to a classical current $j^{\mu}$, then the change in the action by adding one such cosmic string is

$$
\delta S=(1 / q) \int \partial_{\mu} \theta j^{\mu},
$$

which follows from Eq. (2.6). Taking $j^{\mu}$ to be the current of a particle with unit $\mathrm{U}(1)$ charge (and hence nontrivial $Z_{q}$ charge) that circles the string, one finds that

$$
\delta S=\frac{2 \pi}{q} .
$$

This implies an observable Aharanov-Bohm effect in the scattering of particles with discrete gauge charge off cosmic strings. Krauss and Wilczek [18] use this observation to argue that the scattering of a cosmic string off a particle with discrete gauge charge that is falling into a black hole is insensitive to the point at which the particle crosses the event horizon. Thus, the discrete charge of the particle is not lost, and the black hole grows quantum-mechanical hair.

It is interesting to note that the discussion above may be rephrased in unitary gauge by making the initial replacements

$$
B_{\mu}=A_{\mu}-(1 / q) \partial_{\mu} \theta, \quad \text { and } \Phi=e^{-i \theta / q} \phi,
$$

in Eq. (2.2), which then becomes

$$
\begin{aligned}
\mathcal{L}= & -\frac{1}{4 g^{2}} F_{\mu \nu} F^{\mu \nu}+\frac{1}{2} \partial_{\mu} \sigma \partial^{\mu} \sigma+\frac{1}{2}(\Lambda+\sigma)^{2} q^{2} B^{\mu} B_{\mu} \\
& +\left|\partial_{\mu} \Phi-i B_{\mu} \Phi\right|^{2}+V(\sigma) .
\end{aligned}
$$

Unlike the previous approach, all the fields above are gauge invariant; one may integrate out $B_{\mu}$ and $\sigma$, and obtain all the possible $Z_{q}$-invariant interactions involving the light field $\Phi$. This formulation of the low-energy theory is peculiar in that the periodicity of $\theta$ implies that

$$
e^{2 n \pi i / q} \Phi \equiv \Phi, \quad \text { for all integers } n \text {. }
$$

Thus, the field manifold of $\phi$ is not the complex plane $C$, but rather the orbifold $C / Z_{q}$ : Field configurations connected by $Z_{q}$ transformations are identified, and hence are physically redundant, the hallmark of a gauge symmetry. Given this manifold, the field $\Phi$ has a conical singularity at the origin in field space; strings in unitary gauge correspond to $\Phi$ field configurations that wrap around this singularity as the azimuthal angle varies from 0 to $2 \pi$.

As the previous $\mathrm{U}(1) \rightarrow Z_{q}$ example demonstrates, a discrete gauge symmetry can arise in a renormalizable field theory when a continuous gauge symmetry is spontaneously broken by a Higgs field VEV that leaves a discrete symmetry unbroken. The same can occur for non-Abelian symmetries as well. For example, one may break a gauged SU(2) symmetry with a Higgs field transforming as a 7 (which contains a $T^{\prime}$ singlet), leaving the theory invariant under $T^{\prime}$. On the other hand, the $\mathrm{U}(1) \rightarrow Z_{q}$ example suggests how a discrete symmetry may be defined without an explicit embedding in a continuous group. In string theory, the discrete symmetry may be a remnant of general coordinate invariance, ordinary gauge invariance, or the larger gauge symmetry of string theory [17]. For our purposes, however, the nature of the high energy theory is irrelevant.

It is worth mentioning in passing that spontaneouslybroken discrete gauge symmetries have domain walls that are not topologically stable. Holes bounded by strings may spontaneously nucleate, allowing the walls to tear themselves to pieces while dissipating energy through gravita- 


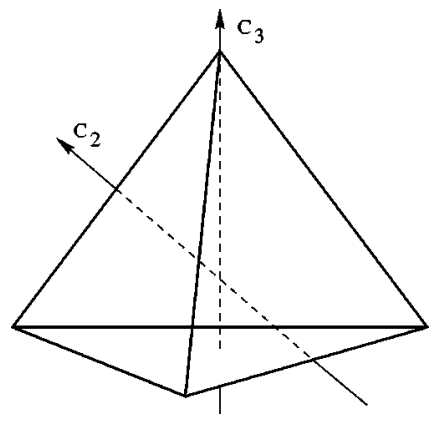

FIG. 1. Geometrical illustration of the group $T^{\prime}$ or $T$. The rotations $C_{2}$ and $C_{3}$ generate all other rotations in each group.

tional radiation [19]. The effectiveness of this mechanism at avoiding cosmological problems is not relevant to our discussion since the flavor-symmetry-breaking scale in our models is high enough (of order the unification scale) that all topological defects are eliminated by inflation.

Finally, it is relevant to consider whether there are any constraints on the low-energy particle content of theories with discrete gauge symmetries. Since continuous gauge symmetries must satisfy anomaly-cancellation conditions, the particle content of low-energy theories with discrete gauge symmetries is restricted. Ibáñez and Ross [20] were the first to consider the constraints on a discrete gauged $Z_{q}$ symmetry, and their results were refined by Banks and Dine [21]: Let $G_{0}$ be a simple factor of the continuous group in which a discrete gauge symmetry is embedded, and let $G_{A}$ and $G_{N}$ represent the unbroken Abelian and non-Abelian gauge symmetries of the low-energy effective theory. Cancellation of the $G_{0} G_{N}^{2}$ anomaly is the only new requirement for consistency of the low-energy theory; all other anomalycancellation constraints involving $G_{0}$ can be satisfied by the introduction of heavy states. Banks and Dine point out that this requirement, termed the linear Ibannez-Ross condition, is equivalent to demanding discrete gauge invariance of nonperturbative interactions generated by instantons of the unbroken continuous gauge groups. This observation demonstrates that consistency of a discrete gauge symmetry at low energies can be established without reference to any particular embedding.

\section{GROUP $T^{\prime}$}

All of the symmetries described in this paper contain $T^{\prime}$, the double tetrahedral group. ${ }^{2}$ Geometrically, $T^{\prime}$ is defined as the group of all 24 proper rotations in three dimensions leaving a regular tetrahedron invariant in the $\mathrm{SU}(2)$ double covering of $\mathrm{SO}(3)$. This perhaps opaque definition may be understood in the following way. There exists a group of 12 elements called the tetrahedral group $T$, consisting of all proper rotations in three dimensions leaving a regular tetrahedron invariant (Fig. 1). It is constructed by parametrizing the group $\mathrm{SO}(3)$ of all proper rotations in three dimensions

${ }^{2}$ For a review of basic terms of discrete group theory, see Ref. [22], Appendix A.
TABLE I. Character table of the double tetrahedral group $T^{\prime}$. The phase $\eta$ is $\exp (2 \pi i / 3)$.

\begin{tabular}{lccccccc}
\hline \hline Sample element & $E$ & $R$ & $C_{2}, C_{2} R$ & $C_{3}$ & $C_{3}^{2}$ & $C_{3} R$ & $C_{3}^{2} R$ \\
\hline Order of class & 1 & 1 & 6 & 4 & 4 & 4 & 4 \\
Order of element & 1 & 2 & 4 & 6 & 3 & 3 & 6 \\
$\mathbf{1}^{0}$ & 1 & 1 & 1 & 1 & 1 & 1 & 1 \\
$\mathbf{1}^{+}$ & 1 & 1 & 1 & $\eta$ & $\eta^{2}$ & $\eta$ & $\eta^{2}$ \\
$\mathbf{1}^{-}$ & 1 & 1 & 1 & $\eta^{2}$ & $\eta$ & $\eta^{2}$ & $\eta$ \\
$\mathbf{2}^{0}$ & 2 & -2 & 0 & 1 & -1 & -1 & 1 \\
$\mathbf{2}^{+}$ & 2 & -2 & 0 & $\eta$ & $-\eta^{2}$ & $-\eta$ & $\eta^{2}$ \\
$\mathbf{2}^{-}$ & 2 & -2 & 0 & $\eta^{2}$ & $-\eta$ & $-\eta^{2}$ & $\eta$ \\
$\mathbf{3}$ & 3 & 3 & -1 & 0 & 0 & 0 & 0 \\
\hline \hline
\end{tabular}

in terms of familiar Euler angles, and then restricting their values to those describing rotations taking a regular tetrahedron into coincidence with itself. The same Euler angles describe rotations in $\mathrm{SU}(2)$ space, since $\mathrm{SU}(2)$ and $\mathrm{SO}(3)$ are locally isomorphic, so that $T^{\prime}$ is the subgroup of $\mathrm{SU}(2)$ corresponding to the same Euler angles as $T \subset \mathrm{SO}(3)$. One therefore expects that even-dimensional representations of $T^{\prime}$ are spinorial, i.e., are multiplied by -1 under a $2 \pi$ rotation (called $R$ in the literature), while odd-dimensional representations of $T^{\prime}$ coincide with those of $T$ and are invariant under this rotation, as may be verified by the character table, Table I.

$T^{\prime}$ is generated by the rotations $C_{2}$ and $C_{3}$ depicted in Fig. 1. Because of the double-valued nature of $T^{\prime}$ rotations, these elements actually have orders 4 and 6 , respectively. For reasons to be described below, it turns out to be convenient to present explicit representations (reps) for an element of order 4 (such as $C_{2}$ ) and one of order 3 (such as $C_{3} R$ ). We label these elements as $g_{5}$ and $g_{9}$, respectively; ${ }^{3}$ then $T^{\prime}$ is defined by the multiplication rules $g_{9}^{3}=g_{5}^{4}=1, g_{9} g_{5}^{2}=g_{5}^{2} g_{9}$, and $g_{5} g_{9}^{-1} g_{5}=g_{9} g_{5} g_{9}$. One may then show that each of the 24 elements may be written uniquely in the canonical form $g_{9}^{p} g_{5}^{q} g_{9}^{r}$, where $p=0, \pm 1$, and if $q=0$ or 2, then $r=0$, while if $q= \pm 1$, then $r=0, \pm 1$.

The group $T^{\prime}$ is central to our model building since it is the smallest with 1-, 2-, and 3-dimensional reps and the multiplication rule $\mathbf{2} \otimes \mathbf{2}=\mathbf{3} \oplus \mathbf{1}$. $T^{\prime}$ models therefore allow for flavons that perform the same roles as $\phi_{a}, S_{a b}$ and $A_{a b}$ in the U(2) model. The only other 24-element group that has reps of the same dimensions is the octahedral group $O$ (which is isomorphic to $S_{4}$ ). In this case, however, the product of two doublet reps does not contain a triplet, and the analogy to $\mathrm{U}(2)$ is lost.

More specifically, $T^{\prime}$ has three singlets $\mathbf{1}^{0}$ and $\mathbf{1}^{ \pm}$, three doublets, $\mathbf{2}^{0}$ and $\mathbf{2}^{ \pm}$, and one triplet, $\mathbf{3}$. The triality superscript provides a concise way of stating the multiplication rules for these reps: With the identification of \pm as \pm 1 ,

\footnotetext{
${ }^{3}$ The element labels are chosen to coincide with those of Thomas and Wood [23], where $T^{\prime}$ is seen to be isomorphic to $\operatorname{SL}_{2}\left(\mathrm{~F}_{3}\right)$, the group of $2 \times 2$ unimodular matrices whose elements are added and multiplied as integers modulo 3 .
} 
trialities add under addition modulo three, and the following rules hold:

$\mathbf{1} \otimes \mathbf{R}=\mathbf{R} \otimes \mathbf{1}=\mathbf{R}$ for any rep $\mathbf{R}, \quad \mathbf{2} \otimes \mathbf{2}=\mathbf{3} \oplus \mathbf{1}$,

\section{$\mathbf{2} \otimes \mathbf{3}=\mathbf{3} \otimes \mathbf{2}=\mathbf{2}^{0} \oplus \mathbf{2}^{+} \oplus \mathbf{2}^{-}, \quad \mathbf{3} \otimes \mathbf{3}=\mathbf{3} \oplus \mathbf{3} \oplus \mathbf{1}^{0} \oplus \mathbf{1}^{+} \oplus \mathbf{1}^{-}$.}

Note that trialities flip sign under Hermitian conjugation, so that $\mathbf{2}^{+} \otimes \mathbf{2}^{-}=\mathbf{3} \oplus \mathbf{1}^{0}$ while $\left(\mathbf{2}^{+}\right)^{\dagger} \otimes \mathbf{2}^{-}=\mathbf{3} \oplus \mathbf{1}^{+}$.

The multiplication of $T^{\prime}$ representations may be made explicit by the use of Clebsch-Gordan matrices. For example, let the fields $\chi$ and $\psi$ be column vectors that transform as $\mathbf{2}^{+}$and $\mathbf{2}^{-}$under $T^{\prime}$, respectively. From the rules above, we know that the product of these reps contains a trivial singlet, the $\mathbf{1}^{0}$, but it is not immediately clear how to construct this representation out of the given fields. Formally, we seek a matrix $M$ such that the product

$$
\chi^{T} M \psi \rightarrow \chi^{T} M \psi
$$

under the transformations $\chi \rightarrow R(g) \chi$ and $\psi \rightarrow R(g) \chi$, where $R$ is a two-dimensional matrix rep, and $g$ runs over all elements of the group. From our earlier discussion, it is only necessary that we consider transformations associated with the defining elements, $g_{5}$ and $g_{9}$, to solve for the form of $M$; in the present case, one finds that $M$ is proportional to the Pauli matrix $\sigma_{2}$. This algebraic procedure is easily generalized to products of other reps. Explicit matrix representations for the generating elements $g_{5}$ and $g_{9}$, as well as the complete set of Clebsch-Gordan matrices for combining $T^{\prime}$ reps are provided in the Appendix. The reader should keep in mind that these Clebsch-Gordan matrices must be taken into account if one is to reproduce the Yukawa textures presented later in this paper. For example, without the factor of $\sigma_{2}$, one might not realize that a VEV in the first component of $\chi$ couples only to the second component of $\psi$.

As mentioned in the Introduction, we also require that our discrete flavor symmetry contain a subgroup that rotates first-generation matter fields by a phase. This subgroup plays the same role as the intermediate $U(1)$ symmetry in the $U(2)$ model, and must forbid all entries in the first row and column of each Yukawa matrix. The smallest discrete subgroup that one might consider is a $Z_{2}$ that flips the sign of all first generation matter fields. Unfortunately, such a transformation leaves the 11 entry of each Yukawa matrix invariant (two sign flips), so that the up and down quarks could, in principle, acquire masses that are too large. A $Z_{3}$ phase rotation, on the other hand, does not lead to the same problem, and a $Z_{3}$ subgroup of $T^{\prime}$ is generated by the element $g_{9}$ defined previously. From the Appendix, we see that the twodimensional representation matrices for the element $g_{9}$ are given by

$$
\begin{gathered}
g_{9}\left(\mathbf{2}^{0}\right)=\left(\begin{array}{cc}
\eta^{2} & 0 \\
0 & \eta
\end{array}\right), \quad g_{9}\left(\mathbf{2}^{+}\right)=\left(\begin{array}{cc}
1 & 0 \\
0 & \eta^{2}
\end{array}\right), \\
g_{9}\left(\mathbf{2}^{-}\right)=\left(\begin{array}{ll}
\eta & 0 \\
0 & 1
\end{array}\right),
\end{gathered}
$$

where $\eta \equiv \exp (2 \pi i / 3)$. If matter fields of the first two generations are assigned to the $\mathbf{2}^{-}$rep, one then obtains the desired phase rotation under the $Z_{3}$ subgroup. This observation is at the heart of the global $T^{\prime}$ model presented in Sec. VII. ${ }^{4}$

As we see below, however, models in which $T^{\prime}$ is free of discrete gauge anomalies are much easier to construct if matter fields are assigned to the $2^{0}$ rep instead. In this case, let us consider extending the flavor symmetry group to $T^{\prime} \times Z_{3}$. We identify a new triality index $0,+$ and - with the $Z_{3}$ phase rotations $1, \eta$, and $\eta^{2}$, respectively. Like the $T^{\prime}$ indices, the $Z_{3}$ trialities also combine via addition modulo 3 . Reps of $T^{\prime} \times Z_{3}$ are denoted by affixing this additional triality as a superscript, e.g., $\mathbf{2}^{+-}$. We now identify the desired intermediate symmetry as the diagonal subgroup of the original $Z_{3}$, generated by the element $g_{9}$, and the new $Z_{3}$ factor. We call this subgroup $Z_{3}^{D}$ henceforth. It is easy to see that the rep $2^{0-}$ transforms under $Z_{3}^{D}$ by the matrix

$$
\left(\begin{array}{ll}
\eta & 0 \\
0 & 1
\end{array}\right)
$$

which is simply the product of $g_{9}\left(\mathbf{2}^{0}\right)$ and $\eta^{2}$. The matter field assignments $\mathbf{2}^{0-} \oplus \mathbf{1}^{00}$, and the breaking pattern $T^{\prime}$ $\times Z_{3} \rightarrow Z_{3}^{D} \rightarrow$ nothing are at the heart of the minimal flavor model discussed in the next section. It is worth pointing out that the reps $\mathbf{1}^{00}, \mathbf{1}^{+-}, \mathbf{1}^{-+}, \mathbf{2}^{0-}, \mathbf{2}^{++}$and $\mathbf{2}^{-0}$ are special in that these singlet reps and the second component of the doublets remain invariant under $Z_{3}^{D}$. Thus any $\mathbf{2} \oplus \mathbf{1}$ combination of these reps is potentially useful in building models with $\mathrm{U}(2)$-like textures.

Finally, we return to the issue of anomaly cancellation. We pointed out in Sec. II that consistency of a discrete gauge symmetry at low energies only requires the cancellation of anomalies that (1) involve the unbroken non-Abelian continuous gauge groups and (2) are linear in a continuous group in which the discrete group is embedded. If we embed $T^{\prime}$ in SU(2), then these constraints are satisfied automatically, providing that the particle content of a given model fills complete $\mathrm{SU}(2)$ representations. Let us therefore consider the embedding of $T^{\prime}$ in $\mathrm{SU}(2)$ in more detail.

The group $\mathrm{SU}(2)$ has one rep of each nonnegative integral dimension $n$ [the spin $(n-1) / 2$ rep], while $T^{\prime}$ has only singlet, doublet, and triplet reps. It must be the case that large $\mathrm{SU}(2)$ reps break up into a number of $T^{\prime}$ reps with the same total dimension. To see this decomposition, consider the characteristic polynomial of matrices in each of the $T^{\prime}$ reps

\footnotetext{
${ }^{4}$ One can also imagine models in which the symmetry group breaks to a non-Abelian subgroup; however, in this case the simple rephasing of multiplet components under the subgroup is not guaranteed.
} 
TABLE II. Decomposition of SU(2) reps into reps of $T^{\prime} . N$ is any non-negative integer.

\begin{tabular}{lc}
\hline \hline SU(2) rep multiplicity & $T^{\prime}$ rep decomposition \\
\hline $12 N$ & $2 N\left\{\mathbf{2}^{0} \oplus \mathbf{2}^{+} \oplus \mathbf{2}^{-}\right\}$ \\
$12 N+1$ & $\mathbf{1}^{0} \oplus N\left\{\mathbf{1}^{0} \oplus \mathbf{1}^{+} \oplus \mathbf{1}^{-} \oplus 3 \cdot \mathbf{3}\right\}$ \\
$12 N+2$ & $\mathbf{2}^{0} \oplus 2 N\left\{\mathbf{2}^{0} \oplus \mathbf{2}^{+} \oplus \mathbf{2}^{-}\right\}$ \\
$12 N+3$ & $\mathbf{3} \oplus N\left\{\mathbf{1}^{0} \oplus \mathbf{1}^{+} \oplus \mathbf{1}^{-} \oplus 3 \cdot \mathbf{3}\right\}$ \\
$12 N+4$ & $\left\{\mathbf{2}^{+} \oplus \mathbf{2}^{-}\right\} \oplus 2 N\left\{\mathbf{2}^{0} \oplus \mathbf{2}^{+} \oplus \mathbf{2}^{-}\right\}$ \\
$12 N+5$ & $\left\{\mathbf{1}^{+} \oplus \mathbf{1}^{-} \oplus \mathbf{3}\right\} \oplus N\left\{\mathbf{1}^{0} \oplus \mathbf{1}^{+} \oplus \mathbf{1}^{-} \oplus 3 \cdot \mathbf{3}\right\}$ \\
$12 N+6$ & $(2 N+1)\left\{\mathbf{2}^{0} \oplus \mathbf{2}^{+} \oplus \mathbf{2}^{-}\right\}$ \\
$12 N+7$ & $\left\{\mathbf{1}^{0} \oplus 2 \cdot \mathbf{3}\right\} \oplus N\left\{\mathbf{1}^{0} \oplus \mathbf{1}^{+} \oplus \mathbf{1}^{-} \oplus 3 \cdot \mathbf{3}\right\}$ \\
$12 N+8$ & $\mathbf{2}^{0} \oplus(2 N+1)\left\{\mathbf{2}^{0} \oplus \mathbf{2}^{+} \oplus \mathbf{2}^{-}\right\}$ \\
$12 N+9$ & $\left\{\mathbf{1}^{0} \oplus \mathbf{1}^{+} \oplus \mathbf{1}^{-} \oplus 2 \cdot \mathbf{3}\right\} \oplus N\left\{\mathbf{1}^{0} \oplus \mathbf{1}^{+} \oplus \mathbf{1}^{-} \oplus 3 \cdot \mathbf{3}\right\}$ \\
$12 N+10$ & $\left\{\mathbf{2}^{+} \oplus \mathbf{2}^{-}\right\} \oplus(2 N+1)\left\{\mathbf{2}^{0} \oplus \mathbf{2}^{+} \oplus \mathbf{2}^{-}\right\}$ \\
$12 N+11$ & $\left.\mathbf{1}^{+} \oplus \mathbf{1}^{-} \oplus 3 \cdot \mathbf{3}\right\} \oplus N\left\{\mathbf{1}^{\mathbf{0}} \oplus \mathbf{1}^{+} \oplus \mathbf{1}^{-} \oplus 3 \cdot \mathbf{3}\right\}$ \\
&
\end{tabular}

for any two rotations that generate the full group. The same can be done for the full SU(2) group restricted to the particular Euler angles that give $T^{\prime}$. Then a large rep matrix of $\mathrm{SU}(2)$ is block diagonalizable into smaller blocks corresponding to rep matrices of $T^{\prime}$; in particular, the characteristic polynomial of the $\mathrm{SU}(2)$ matrix is the product of those of the $T^{\prime}$ matrices. It is then possible to extract which $T^{\prime}$ reps appear in a given $\mathrm{SU}(2)$ rep, as well as their multiplicities. The results of this decomposition are summarized in Table II. There we see that the $\mathbf{1}^{0}, \mathbf{2}^{0}$, and $\mathbf{3}$ reps of $T^{\prime}$ correspond to the complete $\mathbf{1}, \mathbf{2}$, and $\mathbf{3}$ reps of $\mathrm{SU}(2)$. It follows, for example, that $T^{\prime}$ is non-anomalous in all models utilizing the $\mathbf{2}^{0-} \oplus \mathbf{1}^{00}$ representation structure for the matter fields (with Higgs fields as singlets). Note that there is no meaningful low-energy constraint on the $Z_{3}$ charges since Abelian factors may be embedded at high energies in U(1) gauge groups whose anomalies are cancelled by the GreenSchwarz mechanism [14].

\section{MINIMAL MODEL}

In this section we review the minimal $T^{\prime} \times Z_{3}$ model presented in Ref. [12], which we study in quantitative detail in Section $\mathrm{V}$. The three generations of matter fields are assigned to the $T^{\prime} \times Z_{3}$ reps $\mathbf{2}^{0-} \oplus \mathbf{1}^{00}$ while the Higgs fields $H_{U, D}$ are taken to be pure $G_{f}$ singlets. Given these assignments, it is easy to obtain the transformation properties of the Yukawa matrices:

$$
Y_{U, D, L} \sim\left(\begin{array}{cc}
{\left[\mathbf{3}^{-} \oplus \mathbf{1}^{0-}\right]} & {\left[\mathbf{2}^{0+}\right]} \\
{\left[\mathbf{2}^{0+}\right]} & {\left[\mathbf{1}^{00}\right]}
\end{array}\right)
$$

Equation (4.1) indicates the flavon reps needed to construct the fermion mass matrices, namely, $\mathbf{1}^{0-}, \mathbf{2}^{0+}$, and $\mathbf{3}^{-}$, which we call $A, \phi$, and $S$, respectively. Once these flavons acquire VEVs, the flavor group is broken. We are interested in a two-step breaking

$$
T^{\prime} \otimes Z_{3} \stackrel{\epsilon}{\rightarrow} \underset{Z_{3}^{D}}{\epsilon^{\prime}} \rightarrow \text { nothing }
$$

where $\epsilon^{\prime}<\epsilon$ again represent ratios of flavon VEVs to the scale $M_{f}$. Since we have chosen a "special" doublet rep for the first two generations, which transforms as $\operatorname{diag}\{\eta, 1\}$ un$\operatorname{der} Z_{3}^{D}$, only the 22,23 , and 32 entries of the Yukawa matrices may develop VEVs of $O(\epsilon)$ originating from vevs in $S$ and $\phi$. The symmetry $Z_{3}^{D}$ is then broken by a $\mathbf{1}^{0-} \mathrm{VEV}$ of $O\left(\epsilon^{\prime}\right)$. The Clebsch-Gordan coefficient that couples a $\mathbf{1}^{0-}$ to two $2^{0-}$ doublets is proportional to $\sigma_{2}$, so the $\epsilon^{\prime}$ appears in an antisymmetric matrix. These considerations yield the textures

$$
Y_{U, D, L} \sim\left(\begin{array}{ccc}
0 & \epsilon^{\prime} & 0 \\
-\epsilon^{\prime} & \epsilon & \epsilon \\
0 & \epsilon & 1
\end{array}\right),
$$

where $O(1)$ coefficients have been omitted. Since the $\mathbf{1}^{0-}$ and $\mathbf{3}^{-}$flavon VEVs appear as antisymmetric and symmetric matrices, respectively, all features of the grand unified extension of the $\mathrm{U}(2)$ model are obtained here, assuming the same GUT transformation properties are assigned to $\phi, S$, and $A$. One can also show readily that the squark and slepton masssquared matrices are the same as in the U(2) model.

It was shown in Ref. [12] that this simple model can be extended to describe the observed deficit of solar and atmospheric neutrinos. Models for lepton masses were constructed both with and without the assumption of SU(5) unification. The latter possibility is of interest, for example, if one is only concerned with explaining flavor physics of the lepton sector, and is provided for completeness. In either case, the proposed extensions yield viable neutrino textures with naturally large mixing between the second and third generations. Moreover, these extensions do not alter the charged fermion textures, so that all the relations between masses and mixing angles in the $\mathrm{U}(2)$ model are also predictions of $T^{\prime} \times Z_{3}$. We now review the two cases considered in Ref. [12].

Case I: Here we do not assume grand unification, so that all flavons are $\mathrm{SU}(5)$ singlets. We introduce three generations of right-handed neutrinos transforming as

$$
\nu_{R} \sim \mathbf{2}^{0-} \oplus \mathbf{1}^{-+} .
$$


Note that this representation choice differs from that of the other matter fields only in the third generation. Since $\nu_{R}$ are singlets under the standard model gauge groups, introducing a $\mathbf{1}^{-}$field by itself creates no anomaly problems. The neutrino Dirac and Majorana mass matrices then allow flavons that do not contribute to the charged fermion mass matrices. Their transformation properties are given by

$$
M_{L R} \sim\left(\begin{array}{cc}
{\left[\mathbf{3}^{-} \oplus \mathbf{1}^{0-}\right]} & {\left[\mathbf{2}^{+0}\right]} \\
{\left[\mathbf{2}^{0+}\right]} & {\left[\mathbf{1}^{+-}\right]}
\end{array}\right), \quad M_{R R} \sim\left(\begin{array}{cc}
{\left[\mathbf{3}^{-}\right]} & {\left[\mathbf{2}^{+0}\right]} \\
{\left[\mathbf{2}^{+0}\right]} & {\left[\mathbf{1}^{-+}\right]}
\end{array}\right) .
$$

Note that one obtains the same triplet and nontrivial singlet in the upper $2 \times 2$ block as in the charged fermion mass matrices, as well as one of the same flavon doublets, the $\mathbf{2}^{0+}$; the rep $\mathbf{1}^{0-}$ is not present in $M_{R R}$, since Majorana mass matrices are symmetric. In addition we obtain the reps $\mathbf{2}^{+0}$, $\mathbf{1}^{+-}$, and $\mathbf{1}^{-+}$, which did not appear in Eq. (4.1). New flavon fields can now be introduced with these transformation properties, and their effects on the neutrino physics explored. Let us introduce a single ${ }^{5}$ new flavon $\phi_{\nu}$ transforming as a $\mathbf{2}^{+0}$ and with a VEV

$$
\frac{\left\langle\phi_{\nu}\right\rangle}{M_{f}} \sim \sigma_{2}\left(\begin{array}{c}
\epsilon^{\prime} \\
\epsilon
\end{array}\right),
$$

where $\sigma_{2}$ is the Clebsch that couples the two doublets to $\mathbf{1}^{0-}$. This new flavon is the only extension we make to the model in order to describe the neutrino phenomenology. After introducing $\phi_{\nu}$, the neutrino Dirac and Majorana mass matrices read

$$
\begin{aligned}
M_{L R} & \approx\left(\begin{array}{ccc}
0 & l_{1} \epsilon^{\prime} & l_{3} r_{2} \epsilon^{\prime} \\
-l_{1} \epsilon^{\prime} & l_{2} \epsilon & l_{3} r_{1} \epsilon \\
0 & l_{4} \epsilon & 0
\end{array}\right)\left\langle H_{U}\right\rangle, \\
M_{R R} & \approx\left(\begin{array}{ccc}
r_{4} r_{2} \epsilon^{\prime 2} & r_{4} r_{1} \epsilon \epsilon^{\prime} & r_{2} \epsilon^{\prime} \\
r_{4} r_{1} \epsilon \epsilon^{\prime} & r_{3} \epsilon & r_{1} \epsilon \\
r_{2} \epsilon^{\prime} & r_{1} \epsilon & 0
\end{array}\right) \Lambda_{R},
\end{aligned}
$$

where $\Lambda_{R}$ is the right-handed neutrino mass scale, and we have parametrized the $O(1)$ coefficients. Furthermore, the charged lepton Yukawa matrix including $O(1)$ coefficients reads

$$
Y_{L} \approx\left(\begin{array}{ccc}
0 & c_{1} \epsilon^{\prime} & 0 \\
-c_{1} \epsilon^{\prime} & 3 c_{2} \epsilon & c_{3} \epsilon \\
0 & c_{4} \epsilon & c_{5}
\end{array}\right) \xi
$$

The factor of 3 in the 22 entry is simply assumed at present, but originates from the Georgi-Jarlskog mechanism in the grand unified case considered next.

The left-handed Majorana mass matrix $M_{L L}$ follows from the seesaw mechanism

$$
M_{L L} \approx M_{L R} M_{R R}^{-1} M_{L R}^{T},
$$

\footnotetext{
${ }^{5}$ Assuming more than one $\phi_{\nu}$ leads to the same qualitative results.
}

which yields

$$
M_{L L} \sim\left(\begin{array}{ccc}
\left(\epsilon^{\prime} / \epsilon\right)^{2} & \epsilon^{\prime} / \epsilon & \epsilon^{\prime} / \epsilon \\
\epsilon^{\prime} / \epsilon & 1 & 1 \\
\epsilon^{\prime} / \epsilon & 1 & 1
\end{array}\right) \frac{\left\langle H_{U}\right\rangle^{2} \epsilon}{\Lambda_{R}},
$$

where we have suppressed the $O(1)$ coefficients. It is clear by inspection that we naturally obtain large mixing between second- and third-generation neutrinos. It is also important to point out that the two eigenvalues of Eq. (4.10) that appear to be of $O(1)$ depend sensitively on the products of a large number of order one coefficients. It is easy to obtain a hierarchy of order 10 in the two largest mass eigenvalues, without allowing any of the coefficients defined in Eqs. (4.7),(4.8) to deviate from unity by more than a factor of 2 . This comment is important in understanding how the reasonable coefficient choices given in Ref. [12] account for the differing mass scales associated with atmospheric and solar neutrino oscillations.

In order to determine neutrino oscillation parameters precisely one needs to compute the neutrino CKM matrix. If $M_{L L}$ and $Y_{L}$ are diagonalized by $M_{L L}=W M_{L L}^{0} W^{\dagger}, Y_{L}$ $=U_{L} Y_{L}^{0} U_{R}^{\dagger}$, then

$$
V=U_{L}^{\dagger} W
$$

We parametrize this matrix as in Ref. [24]:

V

$$
=\left(\begin{array}{ccc}
c_{12} c_{13} & c_{13} s_{12} & s_{13} \\
-c_{23} s_{12} e^{i \phi}-c_{12} s_{13} s_{23} & c_{12} c_{23} e^{i \phi}-s_{12} s_{13} s_{23} & c_{13} s_{23} \\
s_{23} s_{12} e^{i \phi}-c_{12} c_{23} s_{13} & -c_{12} s_{23} e^{i \phi}-c_{23} s_{12} s_{13} & c_{13} c_{23}
\end{array}\right),
$$

where $c_{i j}\left(s_{i j}\right)$ stands for $\cos \theta_{i j}\left(\sin \theta_{i j}\right)$. Then one finds

$$
\begin{aligned}
& \sin ^{2}\left(2 \theta_{12}\right)=4 \frac{V_{11}^{2} V_{12}^{2}}{\left(V_{11}^{2}+V_{12}^{2}\right)^{2}}, \\
& \sin ^{2}\left(2 \theta_{23}\right)=4 \frac{V_{23}^{2} V_{33}^{2}}{\left(V_{23}^{2}+V_{33}^{2}\right)^{2}} .
\end{aligned}
$$

The observed atmospheric neutrino fluxes may be explained by $\nu_{\mu}-\nu_{\tau}$ mixing if $\sin ^{2} 2 \theta_{23} \geq 0.8$ and $10^{-3} \leq \Delta m_{23}^{2}$ $\lesssim 10^{-2}$, while the solar neutrino deficit may be accommodated by $\nu_{e}-\nu_{\mu}$ mixing assuming the small-angle MikheyevSmirnov-Wolfenstein (MSW) solution $2 \times 10^{-3} \leqslant \sin ^{2} 2 \theta_{12}$ $\leq 10^{-2}$ for $4 \times 10^{-6} \leq \Delta m_{12}^{2} \leq 10^{-5}$, where all squared masses are given in $\mathrm{eV}^{2}[25,26]$. These regions of parameter space are the ones obtained most naturally from our models. ${ }^{6}$

\footnotetext{
${ }^{6}$ The experimental ranges for neutrino mixing parameters follow from a two-neutrino mixing approximation which is valid only if the mixing angle $\theta_{13}<15^{\circ}$ [24]. This condition is satisfied in all our models.
} 
Since $\Lambda_{R}$ is not determined from symmetry considerations, it is only necessary to reproduce $\Delta m_{23}^{2} / \Delta m_{12}^{2}$. In Ref. [12] a choice for the $O(1)$ coefficients can be found that yields neutrino mass ratios and mixing angles falling within the desired ranges given above.

Case II: Here we assume SU(5) unification and that the flavons transform nontrivially under the GUT group, namely, $A \sim \mathbf{1}, S \sim \mathbf{7 5}, \phi \sim \mathbf{1}$, and $\Sigma \sim \mathbf{2 4}$. Note that since $\bar{H} \sim \overline{\mathbf{5}}$, the products $S \bar{H}$ and $A \bar{H}$ transform as a $\overline{\mathbf{4 5}}$ and $\overline{\mathbf{5}}$, respectively, ultimately providing a factor of 3 enhancement in the 22 entry of $Y_{L}$ (the Georgi-Jarlskog mechanism). In addition, two $2^{+0}$ doublets are introduced, $\phi_{\nu 1}$ and $\phi_{\nu 2}$, since the texture obtained for the neutrino masses by adding only one extra doublet is not viable. Both doublets $\phi_{\nu}$ have VEVs of the form displayed in Eq. (4.6). As before, the presence of these two new doublets does not alter the form of any charged fermion Yukawa texture.

The neutrino Dirac and Majorana mass matrices now take the forms

$$
\begin{gathered}
M_{L R} \approx\left(\begin{array}{ccc}
0 & l_{1} \epsilon^{\prime} & l_{5} r_{2} \epsilon^{\prime} \\
-l_{1} \epsilon^{\prime} & l_{2} \epsilon^{2} & l_{3} r_{1} \epsilon \\
0 & l_{4} \epsilon & 0
\end{array}\right)\left\langle H_{U}\right\rangle, \\
M_{R R} \approx\left(\begin{array}{ccc}
r_{3} \epsilon^{\prime 2} & r_{4} \epsilon \epsilon^{\prime} & r_{2} \epsilon^{\prime} \\
r_{4} \epsilon \epsilon^{\prime} & r_{5} \epsilon^{2} & r_{1} \epsilon \\
r_{2} \epsilon^{\prime} & r_{1} \epsilon & 0
\end{array}\right) \Lambda_{R},
\end{gathered}
$$

while the charged lepton mass matrix is the same as in Eq. (4.8). Using Eq. (4.9) one obtains the texture

$$
M_{L L} \sim\left(\begin{array}{ccc}
\left(\epsilon^{\prime} / \epsilon\right)^{2} & \epsilon^{\prime} / \epsilon & \epsilon^{\prime} / \epsilon \\
\epsilon^{\prime} / \epsilon & 1 & 1 \\
\epsilon^{\prime} / \epsilon & 1 & 1
\end{array}\right) \frac{\left\langle H_{U}\right\rangle^{2}}{\Lambda_{R}} .
$$

Again, a viable set of $O(1)$ coefficients may be found in Ref. [12].

While the texture in Eq. (4.16) appears to be the same as the one in Eq. (4.10) (up to an overall factor of $\epsilon$ ), there is in fact an important difference: the $O(1)$ entries in Eq. (4.16) have a vanishing determinant at lowest order. The ratio of the two largest eigenvalues are therefore determined by higher order corrections, which must be taken into account to obtain the correct numerical results. ${ }^{7}$ While the zero determinant is lifted at $O(\epsilon)$ in the superpotential, it is interesting that, in this particular case, a comparable correction comes from $\mathrm{D}$ terms that alter the canonical form of the neutrino kinetic energy:

$$
\int d^{4} \theta\left[\nu_{L}^{\dagger} \nu_{L}+\nu_{L}^{\dagger} B \nu_{L}\right]
$$

\footnotetext{
${ }^{7}$ In fact, the analysis made for the model in case I included higher order terms, which did not contribute in any significant way.
}

Here $B$ is a Hermitian matrix that depends on the flavons in the model. The kinetic terms may be put back into canonical form by the superfield redefinition $\nu_{L} \rightarrow \sqrt{1-B} \nu_{L} \approx(1$ $-B / 2) \nu_{L}$. This in turn leads to a correction to $M_{L L}$ :

$$
M_{L L} \rightarrow M_{L L}-1 / 2\left\{B, M_{L L}\right\} .
$$

Numerically, it is only necessary that we retain the largest elements of $B$ :

$$
B \approx\left(\begin{array}{ccc}
\cdot & \cdot & \cdot \\
\cdot & \cdot & a \epsilon \\
\cdot & a \epsilon & \cdot
\end{array}\right),
$$

which also leads to an $O(\epsilon)$ correction to the determinant discussed above. The parameter $a$ is included in the quantitative analysis of the model presented in the next section.

\section{NUMERICAL ANALYSIS}

The numerical check of the unified $T^{\prime} \times Z_{3}$ model presented in [12] relied on two assumptions. The first is that there exist $O(1)$ coefficients $c_{i}, d_{i}$, and $u_{i}$ for the charged fermion Yukawa matrices that, when combined with the particular choice of neutrino Yukawa parameters $l_{i}$ and $r_{i}$, yield charged fermion mass eigenvalues and mixing angles in agreement with the values observed. This should not be a problem since the textures of the $T^{\prime} \times Z_{3}$ model for the charged fermions agree completely with those of the U(2) model [10], in which all of these observables are accommodated in detailed fits. Second, the textures as written in the last section are defined at the scale $M_{\mathrm{GUT}} \approx 2 \times 10^{16} \mathrm{GeV}$, while the observables are of course measured below the electroweak scale. A truly meaningful fit requires running the gauge and Yukawa couplings over this range. While the textures renormalized at $M_{\mathrm{GUT}}$ and $m_{t}$ should not differ wildly in form, a global fit is required to properly compare the predictions of our model to the experimental data. The purpose of this section is to report on the necessary steps in these fits and the numerical results.

In order to study the renormalization of gauge and Yukawa couplings, we run the one-loop renormalization group equations (RGE's) of the MSSM [27] from $M_{\mathrm{GUT}}$ down to the electroweak scale taken to be $m_{t}=175 \mathrm{GeV}$. This analysis does not include two-loop corrections or threshold effects at either end of the spectrum. In particular, this approach does not differentiate between the scales $M_{f}$, $\epsilon M_{f} \approx M_{\mathrm{GUT}}, \epsilon^{\prime} M_{f} \approx \epsilon^{\prime} M_{\mathrm{GUT}} / \epsilon$, and $\Lambda_{R} \approx \epsilon M_{\mathrm{GUT}} .{ }^{8}$ In any case, both the two-loop and threshold effects are formally of subleading order, and therefore are taken into account by permitting theoretical uncertainties in the gauge and Yukawa couplings of $O\left(1 / 16 \pi^{2}\right) \approx 1 \%$.

Values of the gauge couplings at $M_{\mathrm{GUT}}$ are obtained by starting with the precision values extracted at the scale $M_{Z}$ [28]:

\footnotetext{
${ }^{8}$ Notice that $\Lambda_{R} \approx \epsilon M_{\mathrm{GUT}}$ yields the appropriate mass scale in Eq. (4.16) for atmospheric neutrino oscillations.
} 


$$
\begin{aligned}
& \alpha_{1}^{-1}\left(M_{Z}\right)=59.99 \pm 0.04, \\
& \alpha_{2}^{-1}\left(M_{Z}\right)=29.57 \pm 0.03, \\
& \alpha_{3}^{-1}\left(M_{Z}\right)=8.40 \pm 0.13 .
\end{aligned}
$$

The gauge couplings are run from $M_{Z}$ to $m_{t}$ using the oneloop standard model (SM) RGE's, and then from $m_{t}$ to $M_{\mathrm{GUT}}$ using the one-loop MSSM RGE's. ${ }^{9}$ The GUT scale couplings are taken directly from the textures of Eqs. (1.6), (1.9), (4.8) and (4.15), given numerical values for the dimensionless coefficients $c_{i}, d_{i}, l_{i}, r_{i}, u_{i}$, and $a$ (collectively $\left.k_{i}\right)$, and for $\epsilon, \epsilon^{\prime}, \rho$, and $\xi$. The Yukawa matrices are then run down to $m_{t}$ and diagonalized. ${ }^{10}$

Experimental values for the low-energy Yukawa couplings are extracted from the physical masses and mixing angles compiled by the Particle Data Group [28], where entries of $Y_{U}$ are obtained by dividing quark masses by $v \sin \beta / \sqrt{2}$ and those of $Y_{D, L}$ by dividing quark and lepton masses by $v \cos \beta / \sqrt{2}$, where $v=246 \mathrm{GeV}$.

The experimental uncertainties on the observables (or estimates for the quark masses) used in the fits are either those appearing in Ref. [28] or 1\% of the central value, whichever is larger; since the lepton masses are measured with extraordinary precision, they are sensitive to the two-loop RGE and threshold corrections that we have ignored.

The RGE for the neutrino Majorana mass matrix $M_{L L}$ was computed in Ref. [31] and is included here in order to complete the RGE evolution for all observables. The low-energy neutrino observables are taken to be

$$
\begin{gathered}
100<\frac{\Delta m_{23}^{2}}{\Delta m_{12}^{2}}<2500, \\
\sin ^{2} 2 \theta_{23}>0.8, \\
2 \times 10^{-3}<\sin ^{2} 2 \theta_{12}<10^{-2} .
\end{gathered}
$$

For the sake of having meaningful uncertainties, a parameter whose lower bound is much smaller than its upper bound is converted into its logarithm. Instead of Eq. (5.3), we use

\footnotetext{
${ }^{9}$ It should be pointed out that, while the SM RGE's make use of the modified minimal subtraction ( $\overline{\mathrm{MS}}$ ) scheme, the MSSM RGE's in Ref. [27] make use of the dimensional reduction $\overline{\mathrm{DR}}$ scheme [29], which differ at the matching scale ( $m_{t}$ by our choice) by an amount

$$
\frac{4 \pi}{\alpha_{i}^{\overline{D R}}}=\frac{4 \pi}{\alpha_{i}^{\overline{\mathrm{MS}}}}-\frac{1}{3}\left(C_{A}\right)_{i},
$$

where $C_{A}=\{0,2,3\}$ for $i=1,2,3$.

${ }^{10}$ The RGE's are integrated by means of the Runge-Kutta method with adaptive step size control [30]. The results of this method were cross-checked against the results of using Richardson extrapolation with Bulirsch-Stoer stepping [30] and were found to agree to the limits of the expected accuracy of either solution.
}

$$
\begin{aligned}
\ln \left(\frac{\Delta m_{23}^{2}}{\Delta m_{12}^{2}}\right) & =6.22 \pm 1.61, \\
\sin ^{2} 2 \theta_{23} & =0.9 \pm 0.1, \\
\ln \left(\sin ^{2} 2 \theta_{12}\right) & =-5.41 \pm 0.80 .
\end{aligned}
$$

Summarizing this point, we have discussed the details of how inputs consisting of the gauge couplings at $M_{Z}$ and Yukawa matrix parameters at a high scale are manipulated using one-loop RGE's to produce output values for fermion masses and mixing angles observed at low energy. Of course, the salient question is whether one can find a choice of parameters $k_{i}$, where all of these coefficients are $O(1)$, and yet the output quantities are all in agreement with their observed values. ${ }^{11}$ This is accomplished through a $\chi^{2}$ minimization; thus, the complete simulation consists of choosing a set of parameters $k_{i}$ (relevant at $M_{\mathrm{GUT}}$ ), running the RGE's down to $m_{t}$ and comparing with observation to compute a figure of merit, $\chi^{2}$. If $\chi^{2}$ is too large, the parameters $k_{i}$ are adjusted and the procedure is repeated until convergence of $\chi^{2}$ to a minimum is achieved.

The $\chi^{2}$ function assumes a somewhat nonstandard form. Fermion masses and mixing angles are converted to Yukawa couplings $k_{i}^{\text {expt }} \pm \Delta k_{i}$, and contribute an amount

$$
\Delta \chi^{2}=\left(\frac{k_{i}^{\text {expt }}-k_{i}}{\Delta k_{i}}\right)^{2}
$$

to $\chi^{2}$, as usual. There are 15 observables [ 6 quark masses, 3 quark CKM elements (since $C P$ violation is neglected), 3 lepton masses, 2 neutrino mixing angles, and 1 neutrino mass ratio] and 26 parameters $k_{i}$; on the surface, it seems that the fit is always under-constrained. However, our demand that the parameters $k_{i}$ lie near unity imposes additional restrictions, which we include by adding terms to $\chi^{2}$ of the form

$$
\Delta \chi^{2}=\left(\frac{\ln \left|k_{i}\right|}{\ln 3}\right)^{2}
$$

for each $i$. Thus, the parameters $k_{i}$ are effectively no longer free, but are to be treated analogously to pieces of data, each of which contributes one unit to $\chi^{2}$ if it is as large as 3 or as small as $1 / 3$. The particular choice of 3 for this purpose is, of course, a matter of taste. In effect, the inclusion of such terms renders the parameters $k_{i}$ no longer as true degrees of freedom. On the other hand, they are not true pieces of data either, since a value of say, $k_{i}=0.8$ is just as valid as a value of -1.1 for our purposes. Thus, the value of $\chi_{\min }^{2}$ determining a "good" fit is 15 , since there are 15 pieces of true data and effectively no unconstrained fit parameters.

\footnotetext{
${ }^{11} \mathrm{We}$ also allow for variation of the parameters $\epsilon, \epsilon^{\prime}, \rho$, and $\xi$ by hand, but do not minimize with respect to them. Changes in these parameters are equivalent to redefinitions of the $O(1)$ coefficients, so that they merely set the scale for the other parameters of the fit.
} 
TABLE III. Best fit parameters for the $T^{\prime} \times Z_{3}$ model with $\tan \beta=2$. The minimum $\chi^{2}=2.77$.

\begin{tabular}{|c|c|c|c|c|}
\hline \multicolumn{5}{|c|}{$\epsilon=0.04, \rho=0.08, \quad \epsilon^{\prime}=0.004, \quad \xi=0.017$} \\
\hline$c_{1}=-0.93 \pm 0.01$ & $d_{1}=+1.33 \pm 0.45$ & $l_{1}=+0.85 \pm 0.62$ & $r_{1}=+0.94 \pm 0.84$ & $u_{1}=+0.92 \pm 0.31$ \\
\hline$c_{2}=-0.46 \pm 0.03$ & $d_{2}=-0.81 \pm 0.26$ & $l_{2}=-1.01 \pm 1.11$ & $r_{2}=+1.06 \pm 0.95$ & $u_{2}=+1.48 \pm 0.70$ \\
\hline$c_{3}=-1.02 \pm 1.13$ & $d_{3}=+1.55 \pm 0.67$ & $l_{3}=-0.97 \pm 0.75$ & $r_{3}=+1.03 \pm 1.12$ & $u_{3}=-0.90 \pm 0.91$ \\
\hline$c_{4}=-1.03 \pm 1.15$ & $d_{4}=+1.14 \pm 1.33$ & $l_{4}=-1.09 \pm 1.04$ & $r_{4}=-1.07 \pm 1.05$ & $u_{4}=+1.07 \pm 1.21$ \\
\hline$c_{5}=-0.90 \pm 0.01$ & $d_{5}=-1.29 \pm 0.12$ & $l_{5}=-1.11 \pm 0.79$ & $r_{5}=-0.97 \pm 1.03$ & $u_{5}=+1.84 \pm 0.95$ \\
\hline$a=+0.98 \pm 1.06$ & & & & \\
\hline
\end{tabular}

The numerical minimization is carried out using the MINUIT minimization package. As a cross-check, minimization using Powell's direction set method [30] is carried out to make sure that the same minimum is achieved. Since the topography of the $\chi^{2}$ function is complicated due to the numerous parameters involved, it is important to try a number of initial choices for the input parameters $k_{i}$ in order to have confidence that the minimum obtained is close to global. Once convergence is achieved, a parabolic minimum is assumed and a Hessian matrix is computed in order to gauge uncertainties of the parameters.

Detailed numerical fits show that it is not difficult to find parameters $k_{i}$ that satisfy the constraint $\chi_{\min }^{2}<15$. However, in the $T^{\prime} \times Z_{3}$ model, the ratio $m_{b} / m_{t}$ must be accommodated either by a small value of $\xi$ or a large value of $\tan \beta$. For definiteness, we choose $\tan \beta=2$ as a representative value, and find a best fit with $\chi_{\text {min }}^{2}$ of 2.77 . The complete set of parameters is listed in Table III and a comparison to data appears in Table IV. Note especially that the parameters $\epsilon$,

TABLE IV. Experimental values versus fit central values for observables using the inputs of Table III. Masses are in $\mathrm{GeV}$ and all other quantities are dimensionless. Error bars indicate the larger of experimental or $1 \%$ theoretical uncertainties, as described in the text.

\begin{tabular}{lcc}
\hline \hline Observable & Expt. value & Fit value \\
\hline$m_{u}$ & $(3.3 \pm 1.8) \times 10^{-3}$ & $3.5 \times 10^{-3}$ \\
$m_{d}$ & $(6.0 \pm 3.0) \times 10^{-3}$ & $4.0 \times 10^{-3}$ \\
$m_{s}$ & $0.155 \pm 0.055$ & 0.136 \\
$m_{c}$ & $1.25 \pm 0.15$ & 1.24 \\
$m_{b}$ & $4.25 \pm 0.15$ & 4.25 \\
$m_{t}$ & $173.8 \pm 5.2$ & 170.4 \\
$m_{e}$ & $(5.11 \pm 1 \%) \times 10^{-4}$ & $5.11 \times 10^{-4}$ \\
$m_{\mu}$ & $0.106 \pm 1 \%$ & 0.106 \\
$m_{\tau}$ & $1.78 \pm 1 \%$ & 1.78 \\
$\left|V_{u s}\right|$ & $0.221 \pm 0.004$ & 0.221 \\
$\left|V_{u b}\right|$ & $(3.1 \pm 1.4) \times 10^{-3}$ & $2.3 \times 10^{-3}$ \\
$\left|V_{c b}\right|$ & $(3.9 \pm 0.3) \times 10^{-2}$ & $3.9 \times 10^{-2}$ \\
$\Delta m_{23}^{2} / \Delta m_{12}^{2}$ & $100-2500$ & 526 \\
$\ln \left(\Delta m_{23}^{2} / \Delta m_{12}^{2}\right)$ & $6.22 \pm 1.61$ & 6.27 \\
$\sin ^{2} 2 \theta_{12}$ & $2 \times 10^{-3}-10^{-2}$ & $4.5 \times 10^{-3}$ \\
$\ln \left(\sin ^{2} 2 \theta_{12}\right)$ & $-5.41 \pm 0.80$ & -5.40 \\
$\sin ^{2} 2 \theta_{23}$ & $>0.8$ & 0.90 \\
$\sin ^{2} 2 \theta_{13}$ & - & $1.4 \times 10^{-3}$ \\
\hline \hline
\end{tabular}

$\epsilon^{\prime}$, and $\rho$ are somewhat larger (a factor of 2 or more) than their values in the $\mathrm{U}(2)$ model of Ref. [9], where neutrino physics was not considered. From the excellent $\chi^{2}$, one concludes that the $T^{\prime} \times Z_{3}$ model has little difficulty satisfying all of the required constraints including the naturalness of the coefficients, allowing for the small parameter $\xi$ that distinguishes the scale of $Y_{U}$ from $Y_{D, L}$.

While we have seen that the minimal scenario is extremely successful at reproducing fermion masses and mixing angles, there are nonetheless a number of interesting variant models based on $T^{\prime}$ symmetry. We explore these models in the next three sections.

\section{SU(5) $\times$ U(2) WITH NEITHER SU(5) NOR U(2)}

As discussed in the Introduction, the U(2) model must be embedded in a grand unified theory to reproduce all of the observed quark mass hierarchies. In this section we present a model that does exactly the same, without the need for a GUT, by extending the discrete gauged flavor group to $T^{\prime}$ $\times Z_{6}$. We show that this model explains the ratio $m_{b} / m_{t}$, which is merely parametrized in the $\mathrm{U}(2)$ model (and in our other $T^{\prime}$ models). Before presenting the model we comment on notation. As before, we use the triality superscripts + , -, and 0 for the different representations of $T^{\prime}$. For the $Z_{6}$ reps we now introduce the indices $i=0,1, \ldots, 5$, which combine through addition modulo 6 . For example, $\mathbf{2}^{+4} \otimes \mathbf{1}^{+2}$ $=\mathbf{2}^{-0}$, etc. Since $Z_{6}$ is isomorphic to $Z_{3} \times Z_{2}$, one may view the new flavor symmetry as a $Z_{2}$ extension of the $T^{\prime} \times Z_{3}$ flavor group defined in the model of Sec. IV; denoting the $Z_{2}$ reps by + and - , one identifies

\begin{tabular}{ccc}
$Z_{3}$ & $Z_{2}$ & $Z_{6}$ \\
\hline 0 & + & 0 \\
- & - & 1 \\
+ & + & 2 \\
0 & - & 3 \\
- & + & 4 \\
+ & - & 5 \\
\hline
\end{tabular}

That is, the $Z_{6}$ charge is $2 \times\left(Z_{3}\right.$ charge $)+3$ $\times\left(Z_{2}\right.$ charge $)$ modulo 6 . In the remainder of this section we use the more compact $T^{\prime} \times Z_{6}$ notation.

The three generations of matter fields transform as

$$
Q, U, D \sim \mathbf{2}^{04} \oplus \mathbf{1}^{00},
$$




$$
\begin{aligned}
& L \sim \mathbf{2}^{04} \oplus \mathbf{1}^{+4}, \\
& E \sim \mathbf{2}^{+2} \oplus \mathbf{1}^{-2}, \\
& \nu_{R} \sim \mathbf{2}^{04} \oplus \mathbf{1}^{+1} .
\end{aligned}
$$

The matter fields have transformation properties that differ from those in our previous models, and in particular, the electroweak doublet leptons are no longer anomaly free by themselves. The third-generation $L$ field is assigned to a nontrivial $T^{\prime}$ singlet, the $\mathbf{1}^{+}$, which does not form a complete $\mathrm{SU}(2)$ representation. Given the discussion in Sec. II, the $T^{\prime}$ $\mathrm{SU}(2)_{W}^{2}$ anomaly is not automatically cancelled. However, we remedy this problem by assigning non-trivial transformation properties to the Higgs fields:

$$
H_{U} \sim \mathbf{1}^{00}, \quad H_{D} \sim \mathbf{1}^{-2} .
$$

The fields $H_{D}$ and $L_{3}$ are both electroweak doublets and, as far as the non-Abelian quantum numbers are concerned, form a vector-like pair when $H_{D}$ is a $\mathbf{1}^{-}$under $T^{\prime}$. The remaining fields, $E$ and $\nu_{R}$, do not transform under any unbroken non-Abelian continuous gauge groups and thus their $T^{\prime} \times Z_{6}$ quantum numbers may be assigned freely.

In order to break the flavor symmetry and obtain the fermion mass matrices we introduce the following flavons:

$$
\begin{gathered}
S \sim \mathbf{3}^{0}, \quad A \sim \mathbf{1}^{-0}, \quad \phi \sim \mathbf{2}^{02}, \\
\Delta \sim \mathbf{1}^{+4}, \quad \Delta^{\prime} \sim \mathbf{1}^{-2} .
\end{gathered}
$$

In addition to these flavon fields, we introduce two more in the neutrino sector of the theory. Their transformation properties are such that they do not alter the form of the charged fermion Yukawa textures:

$$
\phi_{\nu} \sim \mathbf{2}^{+3}, \quad \Delta_{\nu} \sim \mathbf{1}^{+1}
$$

Together with $\nu_{R}$, these fields are the only ones that transform nontrivially under the $Z_{2}$ subgroup of $Z_{6}$ (i.e., the only ones with odd $Z_{6}$ charges). Again, we are interested in a two-step breaking:

$$
T^{\prime} \times Z_{6} \stackrel{\epsilon}{\rightarrow} \underset{Z_{3}^{D}}{\epsilon^{\prime}} \rightarrow \text { nothing }
$$

where $Z_{3}^{D}$ is precisely the same subgroup as in the minimal $T^{\prime} \times Z_{3}$ model. Thus, by the same arguments presented in Sec. IV, we obtain the following patterns of VEVs:

$$
\begin{gathered}
\frac{\langle S\rangle}{M_{f}} \sim\left(\begin{array}{ll}
0 & 0 \\
0 & \epsilon
\end{array}\right), \quad \frac{\langle A\rangle}{M_{f}} \sim\left(\begin{array}{cc}
0 & \epsilon^{\prime} \\
-\epsilon^{\prime} & 0
\end{array}\right), \\
\frac{\langle\phi\rangle}{M_{f}} \sim \sigma_{2}\left(\begin{array}{l}
0 \\
\epsilon
\end{array}\right), \quad \frac{\langle\Delta\rangle}{M_{f}} \sim \epsilon, \quad \frac{\left\langle\Delta^{\prime}\right\rangle}{M_{f}} \sim \epsilon, \\
\frac{\left\langle\phi_{\nu}\right\rangle}{M_{f}} \sim \sigma_{2}\left(\begin{array}{l}
\epsilon^{\prime} \\
\epsilon
\end{array}\right), \quad \frac{\left\langle\Delta_{\nu}\right\rangle}{M_{f}} \sim \epsilon .
\end{gathered}
$$

Unlike the minimal model described in the previous two sections, the flavons here contribute to the Yukawa matrices in some cases only at quadratic order

$$
\begin{aligned}
& Y_{U} \sim\left(\begin{array}{cc}
{\left[\mathbf{3}^{4} \oplus \mathbf{1}^{04}\right]} & {\left[\mathbf{2}^{02}\right]} \\
{\left[\mathbf{2}^{02}\right]} & {\left[\mathbf{1}^{00}\right]}
\end{array}\right) \sim\left(\begin{array}{cc}
\Delta S+\Delta A+\phi^{2} & \phi \\
\phi & 1
\end{array}\right) \\
& \approx\left(\begin{array}{ccc}
0 & \epsilon \epsilon^{\prime} & 0 \\
-\epsilon \epsilon^{\prime} & \epsilon^{2} & \epsilon \\
0 & \epsilon & 1
\end{array}\right) \text {, } \\
& Y_{D} \sim\left(\begin{array}{cc}
{\left[\mathbf{3}^{2} \oplus \mathbf{1}^{+2}\right]} & {\left[\mathbf{2}^{+0}\right]} \\
{\left[\mathbf{2}^{+0}\right]} & {\left[\mathbf{1}^{+4}\right]}
\end{array}\right) \sim\left(\begin{array}{cc}
\Delta^{\prime} S+\Delta^{\prime} A & \Delta \phi \\
\Delta \phi & \Delta
\end{array}\right) \\
& \approx\left(\begin{array}{ccc}
0 & \epsilon^{\prime} & 0 \\
-\epsilon^{\prime} & \epsilon & \epsilon \\
0 & \epsilon & 1
\end{array}\right) \epsilon \text {, } \\
& Y_{L} \sim\left(\begin{array}{cc}
{\left[\mathbf{3}^{4} \oplus \mathbf{1}^{04}\right]} & {\left[\mathbf{2}^{-4}\right]} \\
{\left[\mathbf{2}^{-4}\right]} & {\left[\mathbf{1}^{+4}\right]}
\end{array}\right) \sim\left(\begin{array}{cc}
\Delta S+\Delta A+\phi^{2} & \Delta^{\prime} \phi+\Delta_{\nu} \phi_{\nu} \\
\Delta^{\prime} \phi+\Delta_{\nu} \phi_{\nu} & \Delta
\end{array}\right) \\
& \approx\left(\begin{array}{ccc}
0 & \epsilon^{\prime} & \epsilon^{\prime} \\
-\epsilon^{\prime} & \epsilon & \epsilon \\
\epsilon^{\prime} & \epsilon & 1
\end{array}\right) \epsilon .
\end{aligned}
$$

We see that the flavons $\Delta$ and $\Delta^{\prime}$ appear in precisely the right way to recover approximate $\mathrm{SU}(5) \times \mathrm{U}(2)$ textures for $Y_{D}$ and $Y_{L}$, with an additional overall factor of $\epsilon$. The only difference is a relatively uninteresting $\epsilon^{\prime}$ entry in the 13 and 31 elements of $Y_{L}$. Notice that the VEV of the $\Sigma$ field has been replaced by $\langle\Delta\rangle$ in Eq. (6.13). Thus, all important features of the $\mathrm{SU}(5) \times \mathrm{U}(2)$ model are reproduced.

Note that the ratio $m_{b} / m_{t}$, which is experimentally observed to be in the range $0.023 \lesssim m_{b} / m_{t} \lesssim 0.026$, is predicted to be of order $\epsilon \approx 0.02$ for $\tan \beta \approx O(1)$, as can be seen from the ratio of the 33 entries in $Y_{U}$ and $Y_{D}$. This is promising since $\tan \beta \approx O(1)$ is the naive expectation if the Higgs potential is not fine-tuned.

Before proceeding to the analysis of the neutrino sector, a few comments are warranted on the possible supersymmetric contributions to FCNC's in this model. As mentioned in the Introduction, scalar superpartners of the first two generations are exactly degenerate in our models when the flavor symmetry is unbroken. The amount of scalar nondegeneracy at low energies is determined by the order at which flavons contribute to the scalar mass matrices. In the minimal model, the flavons contribute quadratically to the scalar masses of the first two generations, as a consequence of the flavons' nontrivial $Z_{3}$ charges. The scalar mass-squared matrices of the $\mathrm{U}(2)$ model are then reproduced. In the current model, however, the flavon $S$ may contribute linearly, since $\mathbf{3}^{0}$ is in the product of $\left(\mathbf{2}^{04}\right)^{\dagger} \otimes\left(\mathbf{2}^{04}\right)$. The important point is that this effect provides an $O(\epsilon)$ correction to the diagonal entries of the scalar mass matrices. In the fermion mass-eigenstate basis, a Cabibbo-like rotation $\theta_{C} \sim \epsilon^{\prime} / \epsilon$ leads to 12 entries in the scalar mass matrices of order $\epsilon^{\prime} \tilde{m}_{0}^{2}$, where $\tilde{m}_{0}^{2}$ is an av- 
erage scalar mass and $\epsilon^{\prime} \approx 0.004$. Taking into account uncertainty in $O(1)$ coefficients, this result is in marginal agreement with the bounds from $C P$-conserving flavor-changing processes, assuming superpartner masses less than $1 \mathrm{TeV}$ [32]. While bounds from $C P$-violating precesses are generically stronger, the $O(1)$ coefficients have unknown phases that one may simply choose in order to avoid these bounds. Without a firm understanding of the origin of $C P$ violation, saying more about these phases entails a degree of speculation that we choose to avoid. Of course, if scalar superpartners are heavy (as in the "more minimal MSSM" [33]) or flavor universal (as in gauge mediation [1], anomaly mediation [2,3], or Scherk-Swartz mechanism [4]), the current $T^{\prime}$ model is completely safe.

Next we examine the neutrino sector of the model. Given the transformation properties of $\nu_{R}$, we calculate the neutrino Dirac and Majorana mass matrices

$$
\begin{gathered}
M_{L R} \sim\left(\begin{array}{cc}
{\left[\mathbf{3}^{4} \oplus \mathbf{1}^{04}\right]} & {\left[\mathbf{2}^{-1}\right]} \\
{\left[\mathbf{2}^{-4}\right]} & {\left[\mathbf{1}^{+1}\right]}
\end{array}\right) \sim\left(\begin{array}{cc}
\Delta S+\Delta A+\phi^{2} & \Delta \phi_{\nu} \\
\Delta^{\prime} \phi+\Delta_{\nu} \phi_{\nu} & \Delta_{\nu}
\end{array}\right)\left\langle H_{U}\right\rangle \\
\approx\left(\begin{array}{ccc}
0 & l_{1} \epsilon^{\prime} & l_{2} r_{1} \epsilon^{\prime} \\
-l_{1} \epsilon^{\prime} & l_{3} \epsilon & l_{2} r_{3} \epsilon \\
l_{4} \epsilon^{\prime} & l_{5} \epsilon & l_{6}
\end{array}\right) \epsilon\left\langle H_{U}\right\rangle, \\
M_{R R} \sim\left(\begin{array}{cc}
{\left[\mathbf{3}^{4}\right]} & {\left[\mathbf{2}^{-1}\right]} \\
{\left[\mathbf{2}^{-1}\right]} & {\left[\mathbf{1}^{+4}\right]}
\end{array}\right) \sim\left(\begin{array}{cc}
\Delta S & \Delta \phi_{\nu} \\
\Delta \phi_{\nu} & \Delta
\end{array}\right) \Lambda_{R} \\
\approx\left(\begin{array}{ccc}
0 & 0 & r_{1} \epsilon^{\prime} \\
0 & r_{2} \epsilon & r_{3} \epsilon \\
r_{1} \epsilon^{\prime} & r_{3} \epsilon & r_{4}
\end{array}\right) \epsilon \Lambda_{R},
\end{gathered}
$$

where $r_{i}$ and $l_{i}$ are $O(1)$ coefficients. To leading order, the seesaw mechanism gives

$$
M_{L L} \sim\left(\begin{array}{ccc}
\epsilon^{\prime 2} / \epsilon & \epsilon^{\prime} & \epsilon^{\prime} \\
\epsilon^{\prime} & 1 & 1 \\
\epsilon^{\prime} & 1 & 1
\end{array}\right) \frac{\epsilon\left\langle H_{U}\right\rangle^{2}}{\Lambda_{R}} .
$$

Note that the texture in Eq. (6.18) is not changed if higherorder corrections are included that lift the zeros in Eqs. (6.16),(6.17). Following the same procedure as before, we diagonalize $M_{L L}$ and $Y_{L}$ and extract the neutrino masses and mixing angles. A global fit of the parameters in this model can in principle be done; however, we just present a viable set of parameters for simplicity. Using the set of values for the $O(1)$ coefficients in $M_{L L}\left(r_{1}, \ldots, r_{4}, l_{1}, \ldots, l_{6}\right)=(1.0$, $1.0,1.0,-1.0,1.2,1.2,1.3,-1.0,-2.0,1.0)$ and assuming all coefficients in $Y_{L}$ are 1.0 except that of the 22 entry, which we set to 3.0 , we obtain

$$
\frac{\Delta m_{23}^{2}}{\Delta m_{12}^{2}}=125, \quad \sin ^{2} 2 \theta_{12}=3.5 \times 10^{-3}, \quad \sin ^{2} 2 \theta_{23}=0.88 .
$$

This agrees with the allowed ranges described in the previous sections. It is worth mentioning that the texture, Eq.
(6.18), is the same as obtained in Ref. [34], and thus the claim in Ref. [35] that this texture cannot account for solar neutrino oscillations is not correct.

\section{GLOBAL $T^{\prime}$ MODEL}

As pointed out in the Introduction, it is not possible to construct a realistic supersymmetric model with a continuous $\mathrm{SU}(2)$ flavor symmetry if scalar universality is not assumed. The argument is straightforward: The left- and right-handed up quark fields must be embedded in $\mathbf{2} \oplus \mathbf{1}$ representations to maintain the heaviness of the top quark, as well as degeneracy of squarks of the first two generations. Given this assignment, the coupling $Q^{a} U^{b} \epsilon_{a b} H_{u}$ is allowed by the unbroken flavor symmetry, which implies the unacceptable relation $m_{u}=m_{c} \approx m_{t}$. The $T^{\prime}$ model below demonstrates that discrete subgroups of $\mathrm{SU}(2)$ are viable for building models of fermion masses, although they are more dangerous than models with additional Abelian factors, as far as supersymmetric FCNC processes are concerned. We first present the model, and then explain how it evades the problem described above.

The crucial feature that allows one to build a successful $T^{\prime} \times Z_{3}$ model is the existence of a doublet representation $\mathbf{2}^{0-}$, whose first generation component alone rotates by a phase under the $Z_{3}^{D}$ subgroup. This choice is unique in models where $T^{\prime}$ is a discrete gauge symmetry, since the $\mathbf{2}^{0}$ rep is the only doublet that fills a complete $\mathrm{SU}(2)$ representation if we embed $T^{\prime}$ in $\mathrm{SU}(2)$. The 4 of SU(2) decomposes into the reps $\mathbf{2}^{+}$and $\mathbf{2}^{-}$, which implies that each is separately anomalous. While it might still be possible to construct models involving anomaly-free combinations of $\mathbf{2}^{+}$and $\mathbf{2}^{-}$reps, we have found no examples that are particularly compelling. On the other hand, if $T^{\prime}$ is assumed to be a global symmetry, then matter fields can be assigned to any of the doublet representations freely. This provides an opportunity for constructing economical models, as we now demonstrate.

Consider the $Z_{3}$ subgroup of $T^{\prime}$ generated by the element $g_{9}$ that acts on the $\mathbf{2}^{0}$ rep as $\operatorname{diag}\left\{\eta^{2}, \eta\right\}$, with $\eta$ defined as in Sec. III. In the $\mathbf{2}^{-}$rep, this element takes the form $\operatorname{diag}\{\eta, 1\}$, which we identify as the desired phase rotation matrix for matter fields of the first two generations. Given our freedom to assign matter fields to any of the doublet reps in a global $T^{\prime}$ model, it is no longer necessary to extend the flavor symmetry by an Abelian factor in order to find a subgroup that forbids the order $\epsilon^{\prime}$ Yukawa entries. Thus, one is naturally led to the charge assignment

$$
\psi \sim \mathbf{2}^{-} \oplus \mathbf{1}^{0} \text { for } \psi=Q, U, D, L \text { and } E \text {, }
$$

and $H_{U, D} \sim \mathbf{1}^{0}$, which yields

$$
Y_{U, D, L} \sim\left(\begin{array}{cc}
{\left[\mathbf{3} \oplus \mathbf{1}^{-}\right]} & {\left[\mathbf{2}^{+}\right]} \\
{\left[\mathbf{2}^{+}\right]} & {\left[\mathbf{1}^{0}\right]}
\end{array}\right) .
$$

Introducing flavons, $A, \phi$ and $S$ transforming as $\mathbf{1}^{-}, \mathbf{2}^{+}$, and $\mathbf{3}$, respectively, one reproduces the canonical $\mathrm{U}(2)$ textures assuming the breaking pattern 


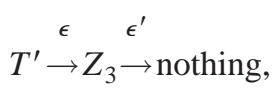

together with the dynamical assumption that only the $\mathbf{1}^{-}$rep participates in the last step of symmetry breaking. The resulting textures are identical to those in our original model of Sec. IV. One difference, however, is that the $S$ flavon in this model contributes to the squark mass matrices at first order in $\epsilon$, just as in the $T^{\prime} \times Z_{6}$ model. However, this is not a concern for the same reasons discussed at length in Sec. VI.

Turning to neutrino physics, recall that successful results were obtained in the $T^{\prime} \times Z_{3}$ model by altering the charge assignment of the third-generation right-handed neutrino field. Thus, we are motivated here to consider

$$
\nu_{R} \sim \mathbf{2}^{-} \oplus \mathbf{1}^{-},
$$

which implies

$$
M_{L R} \sim\left(\begin{array}{cc}
{\left[\mathbf{3} \oplus \mathbf{1}^{-}\right]} & {\left[\mathbf{2}^{-}\right]} \\
{\left[\mathbf{2}^{+}\right]} & {\left[\mathbf{1}^{+}\right]}
\end{array}\right), \quad M_{R R} \sim\left(\begin{array}{cc}
{[\mathbf{3}]} & {\left[\mathbf{2}^{-}\right]} \\
{\left[\mathbf{2}^{-}\right]} & {\left[\mathbf{1}^{-}\right]}
\end{array}\right) .
$$

We identify the flavon $\phi_{\nu}$ with the representation $\mathbf{2}^{-}$, which does not appear in any of the charged fermion Yukawa textures. However, there is an important difference between this model and the one discussed in Sec. IV: The third generation $\nu_{R}$ field transforms by a phase under the $Z_{3}$ subgroup, so that, for example, the 13 and 31 entries of $M_{R R}$ are left invariant under this intermediate symmetry. This implies an inversion in the hierarchy of vevs in the third row and column of $M_{R R}$. In the non-unified version of the model, it is somewhat remarkable that we still obtain a viable form for $M_{L L}$ :

$$
\begin{gathered}
M_{L R} \approx\left(\begin{array}{ccc}
0 & l_{1} \epsilon^{\prime} & l_{5} r_{1} \epsilon \\
-l_{1} \epsilon^{\prime} & l_{2} \epsilon & l_{3} r_{2} \epsilon^{\prime} \\
0 & l_{4} \epsilon & 0
\end{array}\right)\left\langle H_{U}\right\rangle, \\
M_{R R} \approx\left(\begin{array}{ccc}
0 & 0 & r_{1} \epsilon \\
0 & r_{3} \epsilon & r_{2} \epsilon^{\prime} \\
r_{1} \epsilon & r_{2} \epsilon^{\prime} & r_{4} \epsilon^{\prime}
\end{array}\right) \Lambda_{R}, \\
M_{L L} \sim\left(\begin{array}{ccc}
\left(\epsilon^{\prime} / \epsilon\right)^{2} & \epsilon^{\prime} / \epsilon & \epsilon^{\prime} / \epsilon \\
\epsilon^{\prime} / \epsilon & 1 & 1 \\
\epsilon^{\prime} / \epsilon & 1 & 1
\end{array}\right) \frac{\left\langle H_{U}\right\rangle^{2} \epsilon}{\Lambda_{R}} .
\end{gathered}
$$

Unfortunately, this result does not persist in the simplest unified version of the model, which includes additional suppression factors in the 22 entries of $M_{L R}$ and $M_{R R}$. Fortunately, a simple modification of the flavon charge assignments in the unified theory allows us to recover the previous result. We introduce two $\phi_{\nu}$ flavons that transform differently under $T^{\prime} \times \mathrm{SU}(5)$ :

$$
\phi_{\nu} \sim\left(2^{-}, 24\right), \quad \phi_{\nu}^{\prime} \sim\left(2^{-}, \mathbf{1}\right)
$$

Furthermore, we assume the pattern of VEVs:

$$
\left\langle\phi_{\nu}\right\rangle \sim\left(\begin{array}{c}
0 \\
\epsilon
\end{array}\right), \quad\left\langle\phi_{\nu}^{\prime}\right\rangle \sim\left(\begin{array}{c}
\epsilon^{\prime} \\
0
\end{array}\right)
$$

This is consistent with the breaking pattern in Eq. (7.3), but includes a dynamical assumption that the doublet $\phi_{\nu}^{\prime}$ does not participate in the first stage of sequential symmetry breaking and its second component acquires no VEV. ${ }^{12}$ Since $\phi_{\nu}$ transforms as an SU(5) adjoint, it can contribute directly to $M_{L R}$, but only to $M_{R R}$ if, for example, the adjoint flavon $\Sigma$ is also present; the corresponding entries of $M_{R R}$ are therefore suppressed by an additional factor of $\epsilon$ :

$$
\begin{gathered}
M_{L R} \approx\left(\begin{array}{ccc}
0 & l_{1} \epsilon^{\prime} & l_{5} r_{1} \epsilon \\
-l_{1} \epsilon^{\prime} & l_{2} \epsilon^{2} & l_{3} r_{2} \epsilon^{\prime} \\
0 & l_{4} \epsilon & 0
\end{array}\right)\left\langle H_{U}\right\rangle, \\
M_{R R} \approx\left(\begin{array}{ccc}
0 & 0 & r_{1} \epsilon^{2} \\
0 & r_{3} \epsilon^{2} & r_{2} \epsilon^{\prime} \\
r_{1} \epsilon^{2} & r_{2} \epsilon^{\prime} & r_{4} \epsilon^{\prime}
\end{array}\right) \Lambda_{R} .
\end{gathered}
$$

The seesaw mechanism then yields

$$
M_{L L} \sim\left(\begin{array}{ccc}
\left(\epsilon^{\prime} / \epsilon\right)^{2} & \epsilon^{\prime} / \epsilon & \epsilon^{\prime} / \epsilon \\
\epsilon^{\prime} / \epsilon & 1 & 1 \\
\epsilon^{\prime} / \epsilon & 1 & 1
\end{array}\right) \frac{\left\langle H_{U}\right\rangle^{2} \epsilon}{\Lambda_{R}}
$$

where we used the numerical fact that $\epsilon^{\prime 2} / \epsilon^{3} \sim O(1)$. It is important to note that we have only displayed the contributions to Eq. (7.10) linear in $\phi, S$ and $A$, for convenience; quadratic and higher order corrections lift the zero entries of these textures, but do not change the result in Eq. (7.11) qualitatively. Note that Eq. (7.11) is the same successful texture obtained in our original $T^{\prime} \times Z_{3}$ model.

Finally, we return to the no-go theorem presented at the beginning of this section. It is not possible to construct a realistic model with a continuous SU(2) flavor symmetry and $\mathbf{2} \oplus \mathbf{1}$ rep structure because an unwanted flavor-invariant operator may be formed from the product of two doublet matter fields. In our global $T^{\prime}$ model we have the freedom to assign matter fields to new doublet representations whose products contain no trivial singlets, thus avoiding the problem.

\section{VIII. $T^{\prime}$ WITH STERILE NEUTRINOS}

In this section we comment briefly on the possibility of four light neutrino species. Rather than investigating the (vast) space of possible models, we simply show how the results of a successful extension of the $U(2)$ model with a sterile neutrino proposed by Hall and Weiner (HW) [35] can be reproduced with $T^{\prime}$ symmetry instead.

Consider a U(2) model with all matter fields, including

\footnotetext{
${ }^{12} \mathrm{We}$ consistently assume that a flavon that transforms nontrivially under a subgroup $H_{i}$ either acquires a VEV of the order of the scale at which $H_{i}$ is spontaneously broken or acquires no VEV at all.
} 
three generations of right-handed neutrinos, in $\mathbf{2} \oplus \mathbf{1}$ representations. Given the canonical pattern of flavon VEVs, one obtains a right-handed neutrino mass matrix of the form

$$
M_{R R}=\left(\begin{array}{ccc}
0 & 0 & 0 \\
0 & \epsilon & \epsilon \\
0 & \epsilon & 1
\end{array}\right) \Lambda_{R}
$$

Since $M_{R R}$ is symmetric, there is no contribution from the flavon $A$, leading to a singular matrix. It is important to emphasize that the zero entries of Eq. (8.1) are not lifted at any order in $\epsilon$ and $\epsilon^{\prime}$ as a consequence of the holomorphicity of the superpotential. From consideration of the U(2) indices of the flavon fields [or alternatively their charges under a U(1) subgroup of $U(2)$, it is possible to show that any contribution to the vanishing entries of Eq. (8.1) requires the complex conjugation of a flavon field, which is not allowed by unbroken supersymmetry. If the pattern of flavon VEVs is not altered, the first-generation right-handed neutrino remains in the low-energy theory as a sterile neutrino.

This sterile neutrino mixes with the second-generation left-handed neutrino at order $\epsilon^{\prime}$ in $M_{L R}$. After integrating out the two heavy right-handed neutrino flavors, one obtains a four-by-four neutrino mass matrix of the form

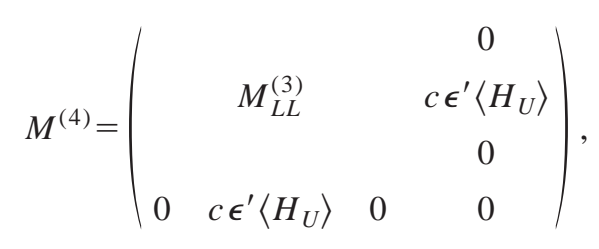

where the three-by-three block $M_{L L}^{(3)}$ has entries of order $\left\langle H_{U}\right\rangle^{2} / \Lambda_{R}$, which can be found in Ref. [35]. HW observe that the 24 and 42 entries of $M^{(4)}$ are much larger than all others, leading naturally to maximal mixing between $\nu_{\mu}$ and the sterile neutrino. As it stands, however, both would have masses of order of the electroweak scale unless $c$ is taken to be of $O\left(10^{-8}\right)$. To obtain a viable model, HW extend the flavor symmetry by an additional U(1) factor, under which all the right-handed neutrinos have charge +1 . A charge -1 flavon is introduced with the $\mathrm{VEV} \epsilon_{N} \sim 10^{-8}$, which breaks this symmetry weakly. One then finds that $c \approx \epsilon_{N}$, while $M^{(3)}$ remains unchanged.

The main obstacle to implementing this solution in a $T^{\prime}$ $\times Z_{3}$ model with all matter fields assigned to $2^{0-} \oplus \mathbf{1}^{00}$ reps is that higher-order corrections to the first row and column of Eq. (8.1) are not forbidden by holomorphicity; the complex conjugate of any non-trivial $Z_{3}$ phase rotation is the same as its square. Thus, we are led to promote our $Z_{3}$ symmetry to a continuous $\mathrm{U}(1) .{ }^{13}$ The appropriate embedding is given by

$$
\psi \sim \mathbf{2}^{0-} \oplus \mathbf{1}^{00} \rightarrow \mathbf{2}_{+1}^{0} \oplus \mathbf{1}_{0}^{0}
$$

\footnotetext{
${ }^{13}$ We could also promote $Z_{3}$ to a much larger $Z_{n}$ that adequately suppresses corrections to the zero entries in Eq. (8.1); we leave this possibility implicit in our discussion.
}

$$
\phi \sim \mathbf{2}^{0+} \rightarrow \mathbf{2}_{-1}^{0}, \quad S \sim \mathbf{3}^{-} \rightarrow \mathbf{3}_{-2}, \quad A \sim \mathbf{1}^{0-} \rightarrow \mathbf{1}_{-2}^{0},
$$

where the subscript indicates the $\mathrm{U}(1)$ charge. Assuming the breaking pattern

$$
T^{\prime} \times U(1) \stackrel{\epsilon}{\rightarrow} \underset{3}{\stackrel{\epsilon^{\prime}}{\longrightarrow}} \rightarrow \text { nothing }
$$

we reproduce the textures of the U(2) model, including Eq. (8.1), identically. The HW predictions for solar, atmospheric and Liquid Scintillation Neutrino Detector (LSND) [36] neutrino oscillations are then recovered by extending the symmetry by an additional U(1) factor, implemented precisely as before. We are thus able to reproduce the results of Ref. [35] with the flavor symmetry $T^{\prime} \times \mathrm{U}(1)^{2}$. Although we find this model less compelling than the other three already discussed, it may be of some relevance if the LSND oscillation result is independently confirmed.

\section{CONCLUSIONS}

We have shown in this paper how to reproduce the quark and charged lepton Yukawa textures of the U(2) model using a minimal non-Abelian discrete symmetry, the double tetrahedral group $T^{\prime}$. The first model we discuss, based on the discrete gauge symmetry $T^{\prime} \times Z_{3}$, not only successfully accommodates the observed charged fermion masses and CKM angles, but also accounts for solar (small-angle MSW) and atmospheric neutrino oscillations. In particular, a large $\nu_{\mu}-\nu_{\tau}$ mixing angle is predicted in the model, even though all charged fermion Yukawa textures are hierarchical. A global fit including neutrino parameters was performed in a grand unified version of the model, and results with extremely good $\chi^{2}$ were obtained.

In addition, two variant $T^{\prime}$ models were discussed. In the first, the flavor group was extended to $T^{\prime} \times Z_{6}$, and all important features of the $\mathrm{SU}(5) \times \mathrm{U}(2)$ model were reproduced without the need for a field-theoretic unification. This model provided a successful prediction (with order-1 uncertainty) of the bottom to top quark Yukawa coupling ratio, which is merely parametrized in the $\mathrm{U}(2)$ model and in the other $T^{\prime}$ models we discuss. The second variant theory was based on a global $T^{\prime}$ symmetry and demonstrates that the successful $\mathrm{U}(2)$ textures can be obtained without including an Abelian factor in the flavor group. In both variant models, large $\nu_{\mu}-\nu_{\tau}$ mixing is predicted, and solutions to the solar and atmospheric neutrino problems are naturally obtained.

It is worth pointing out that the viable neutrino textures predicted by our models are achieved without altering the predictive textures of the charged fermions and without introducing sterile neutrinos. Interestingly, the solutions we present have no simple analogy in the U(2) model: the righthanded neutrino fields in our models do not fill complete $\mathrm{U}(2)$ representations. In particular, the third generation $\nu_{R}$ transforms as a $\mathbf{1}^{-}$, which forms only part of a $\mathbf{5}$ in $\mathrm{U}(2)$. Aside from the possibility of very nonminimal $\mathrm{U}(2)$ models (e.g. with seven generations of right-handed neutrinos), the desired neutrino $T^{\prime}$ reps do not naturally occur. The key 
advantage of discrete groups is that the large, phenomenologically unused representations of the continuous embedding group break up into sets of small phenomenologically useful representations of the discrete group. If discrete gauge symmetries arise as fundamental symmetries of nature, then we see from the example of $T^{\prime}$ that their richer representation structure makes it possible to construct simple and elegant models of flavor.

\section{ACKNOWLEDGMENTS}

A.A. and C.D.C. thank the National Science Foundation for support under Grants Nos. PHY-9800741 and PHY9900657, and the Jeffress Memorial Trust for support under Grant No. J-532. R.F.L. thanks the Department of Energy for support under Contract No. DE-AC05-84ER40150.

\section{APPENDIX: EXPLICIT DETAILS OF T}

As described in the text, the group $T^{\prime}$ is generated by the elements labeled $g_{5}$ and $g_{9}$. We begin by exhibiting explicit matrices representing these elements in each of the seven reps listed in Table I. The singlets are $g_{5}\left(\mathbf{1}^{0, \pm}\right)=1, g_{9}\left(\mathbf{1}^{0}\right)$ $=1, g_{9}\left(\mathbf{1}^{+}\right)=\eta, g_{9}\left(\mathbf{1}^{-}\right)=\eta^{2}$, where $\eta=\exp (2 \pi i / 3)$. The doublets are

$$
\begin{aligned}
& g_{5}\left(\mathbf{2}^{0, \pm}\right)=M_{1}, \quad g_{9}\left(\mathbf{2}^{0}\right)=\eta M_{2}, \\
& g_{9}\left(\mathbf{2}^{+}\right)=\eta^{2} M_{2}, \quad g_{9}\left(\mathbf{2}^{-}\right)=M_{2},
\end{aligned}
$$

where

$$
M_{1}=-\frac{1}{\sqrt{3}}\left(\begin{array}{cc}
+i & +\sqrt{2} e^{i \pi / 12} \\
-\sqrt{2} e^{-i \pi / 12} & -i
\end{array}\right), M_{2}=\left(\begin{array}{ll}
\eta & 0 \\
0 & 1
\end{array}\right),
$$

and the triplet rep is generated by

$$
g_{5}(\mathbf{3})=\frac{1}{3}\left(\begin{array}{ccc}
-1 & 2 \eta & 2 \eta^{2} \\
2 \eta^{2} & -1 & 2 \eta \\
2 \eta & 2 \eta^{2} & -1
\end{array}\right), \quad g_{9}(\mathbf{3})=\left(\begin{array}{ccc}
1 & 0 & 0 \\
0 & \eta & 0 \\
0 & 0 & \eta^{2}
\end{array}\right) \text {. }
$$

The Clebsch-Gordan (CG) coefficient matrices $\mathcal{O}_{i}$ coupling an $n_{x}$-plet $\mathbf{x}$ and an $n_{y}$-plet $\mathbf{y}$ to form an $n_{z}$-plet $\mathbf{z}$ consist of $n_{z}$ matrices of dimensions $n_{x} \times n_{y}$ satisfying the condition

$$
R_{x}^{T} \mathcal{O}_{i} R_{y}=\sum_{j=1}^{n_{z}}\left(R_{z}\right)_{i j} \mathcal{O}_{j}, \quad i=1, \ldots, n_{z},
$$

where $R_{i}$ denotes the group rotation $R$ in rep $i$. In a perhaps more familiar notation, the CGs above may be written

$$
\left(\mathcal{O}_{i}\right)_{j k}=\left(\begin{array}{cc|c}
\mathbf{x} & \mathbf{y} & \mathbf{z} \\
j & k & i
\end{array}\right) .
$$

Note from Eq. (A5) that the CG matrices for $\mathbf{R}_{1} \otimes \mathbf{R}_{2}$ are simply the transposes of those for $\mathbf{R}_{2} \otimes \mathbf{R}_{1}$, and thus are omitted below. The coefficients $c$ below indicate multiplicative constants arbitrary in the definition Eq. (A4). The CG coef- ficients for two singlet reps or any rep with $\mathbf{1}^{0}$ are all unity; the remaining CGs for products involving singlets are

$$
\begin{aligned}
\mathbf{1}^{t_{1} \otimes \mathbf{2}^{t_{2}}=} & \mathbf{2}^{t_{1}+t_{2}}, \quad \text { with } \quad \mathcal{O}_{1}=c\left(\begin{array}{lll}
1 & 0
\end{array}\right), \quad \mathcal{O}_{2}=c\left(\begin{array}{ll}
0 & 1
\end{array}\right), \\
& \mathbf{1}^{+} \otimes \mathbf{3}=\mathbf{3}, \quad \text { with } \quad \mathcal{O}_{1}=c\left(\begin{array}{lll}
0 & 0 & 1
\end{array}\right), \\
& \mathcal{O}_{2}=c\left(\begin{array}{lll}
1 & 0 & 0
\end{array}\right), \quad \mathcal{O}_{3}=c\left(\begin{array}{lll}
0 & 1 & 0
\end{array}\right), \\
& \mathbf{1}^{-} \otimes \mathbf{3}=\mathbf{3}, \quad \text { with } \quad \mathcal{O}_{1}=c\left(\begin{array}{lll}
0 & 1 & 0
\end{array}\right), \\
& \mathcal{O}_{2}=c\left(\begin{array}{lll}
0 & 0 & 1
\end{array}\right), \quad \mathcal{O}_{3}=c\left(\begin{array}{lll}
1 & 0 & 0
\end{array}\right) .
\end{aligned}
$$

Next, let

$$
\begin{gathered}
M_{3}=\frac{1}{2}(1-i)\left(\begin{array}{ll}
0 & 1 \\
1 & 0
\end{array}\right), \quad M_{4}=\left(\begin{array}{ll}
i & 0 \\
0 & 0
\end{array}\right), \\
M_{5}=\left(\begin{array}{ll}
0 & 0 \\
0 & 1
\end{array}\right), \quad M_{6}=\left(\begin{array}{cc}
0 & 1 \\
-1 & 0
\end{array}\right) .
\end{gathered}
$$

Then

$$
\begin{gathered}
\mathbf{2}^{0} \otimes \mathbf{2}^{0} \supset \mathbf{3}, \quad \mathbf{2}^{ \pm} \otimes \mathbf{2}^{\mp} \supset \mathbf{3}: \\
\mathcal{O}_{1}=c M_{3}, \quad \mathcal{O}_{2}=c M_{4}, \quad \mathcal{O}_{3}=c M_{5}, \\
\mathbf{2}^{0} \otimes \mathbf{2}^{0} \supset \mathbf{1}^{0}, \quad \mathbf{2}^{ \pm} \otimes \mathbf{2}^{\mp} \supset \mathbf{1}^{0}: \\
\mathcal{O}=c M_{6}, \\
\mathbf{2}^{0} \otimes \mathbf{2}^{+} \supset \mathbf{3}, \quad \mathbf{2}^{-} \otimes \mathbf{2}^{-} \supset \mathbf{3}: \\
\mathcal{O}_{1}=c M_{5}, \quad \mathcal{O}_{2}=c M_{3}, \quad \mathcal{O}_{3}=c M_{4}, \\
\mathbf{2}^{0} \otimes \mathbf{2}^{+} \supset \mathbf{1}^{+}, \quad \mathbf{2}^{-} \otimes \mathbf{2}^{-} \supset \mathbf{1}^{+}: \\
\mathcal{O}=c M_{6}, \\
\mathbf{2}^{0} \otimes \mathbf{2}^{-} \supset \mathbf{3}, \quad \mathbf{2}^{+} \otimes \mathbf{2}^{+} \supset \mathbf{3}: \\
\mathcal{O}_{1}=c M_{4}, \quad \mathcal{O}_{2}=c M_{5}, \quad \mathcal{O}_{3}=c M_{3}, \\
\mathbf{2}^{0} \otimes \mathbf{2}^{-} \supset \mathbf{1}^{-}, \quad \mathbf{2}^{+} \otimes \mathbf{2}^{+} \supset \mathbf{1}^{-}: \\
\mathcal{O}=c M_{6} .
\end{gathered}
$$

The remaining combinations are

$$
\begin{aligned}
\mathcal{O}_{1}= & c\left(\begin{array}{ccc}
1 & 0 & 0 \\
0 & 1+i & 0
\end{array}\right), \quad \mathcal{O}_{2}=c\left(\begin{array}{ccc}
0 & 0 & 1-i \\
-1 & 0 & 0
\end{array}\right), \\
& \mathbf{2}^{0} \otimes \mathbf{3} \supset \mathbf{2}^{+}, \quad \mathbf{2}^{+} \otimes \mathbf{3} \supset \mathbf{2}^{-}, \quad \mathbf{2}^{-} \otimes \mathbf{3} \supset \mathbf{2}^{0}: \\
\mathcal{O}_{1}= & c\left(\begin{array}{ccc}
0 & 1 & 0 \\
0 & 0 & 1+i
\end{array}\right), \quad \mathcal{O}_{2}=c\left(\begin{array}{ccc}
1-i & 0 & 0 \\
0 & -1 & 0
\end{array}\right),
\end{aligned}
$$




$$
\begin{aligned}
& \mathbf{2}^{0} \otimes \mathbf{3} \supset \mathbf{2}^{-}, \quad \mathbf{2}^{+} \otimes \mathbf{3} \supset \mathbf{2}^{0}, \quad \mathbf{2}^{-} \otimes \mathbf{3} \supset \mathbf{2}^{+}: \\
& \mathcal{O}_{1}=c\left(\begin{array}{ccc}
0 & 0 & 1 \\
1+i & 0 & 0
\end{array}\right), \quad \mathcal{O}_{2}=c\left(\begin{array}{ccc}
0 & 1-i & 0 \\
0 & 0 & -1
\end{array}\right) \\
& \mathbf{3} \otimes \mathbf{3} \supset \mathbf{3}_{s} \oplus \mathbf{3}_{a}: \\
& \mathcal{O}_{1}=c_{1}\left(\begin{array}{ccc}
2 & 0 & 0 \\
0 & 0 & -1 \\
0 & -1 & 0
\end{array}\right)+c_{2}\left(\begin{array}{ccc}
0 & 0 & 0 \\
0 & 0 & -1 \\
0 & 1 & 0
\end{array}\right) \text {, } \\
& \mathcal{O}_{2}=c_{1}\left(\begin{array}{ccc}
0 & -1 & 0 \\
-1 & 0 & 0 \\
0 & 0 & 2
\end{array}\right)+c_{2}\left(\begin{array}{ccc}
0 & -1 & 0 \\
1 & 0 & 0 \\
0 & 0 & 0
\end{array}\right)
\end{aligned}
$$$$
\mathcal{O}_{3}=c_{1}\left(\begin{array}{ccc}
0 & 0 & -1 \\
0 & 2 & 0 \\
-1 & 0 & 0
\end{array}\right)+c_{2}\left(\begin{array}{ccc}
0 & 0 & 1 \\
0 & 0 & 0 \\
-1 & 0 & 0
\end{array}\right) \text {, }
$$

$$
\begin{aligned}
& \mathbf{3} \otimes \mathbf{3} \supset \mathbf{1}^{0}: \quad \mathcal{O}=c\left(\begin{array}{ccc}
1 & 0 & 0 \\
0 & 0 & 1 \\
0 & 1 & 0
\end{array}\right), \\
& \mathbf{3} \otimes \mathbf{3} \supset \mathbf{1}^{+}: \quad \mathcal{O}=c\left(\begin{array}{ccc}
0 & 1 & 0 \\
1 & 0 & 0 \\
0 & 0 & 1
\end{array}\right), \\
& \mathbf{3} \otimes \mathbf{3} \supset \mathbf{1}^{-}: \quad \mathcal{O}=c\left(\begin{array}{ccc}
0 & 0 & 1 \\
0 & 1 & 0 \\
1 & 0 & 0
\end{array}\right) .
\end{aligned}
$$

[1] M. Dine and A.E. Nelson, Phys. Rev. D 48, 1277 (1993); M. Dine, A.E. Nelson, and Y. Shirman, ibid. 51, 1362 (1995).

[2] L. Randall and R. Sundrum, Nucl. Phys. B557, 79 (1999).

[3] G.F. Giudice, M.A. Luty, H. Murayama, and R. Rattazzi, J. High Energy Phys. 12, 027 (1998).

[4] I. Antoniadis, S. Dimopoulos, A. Pomarol, and M. Quiros, Nucl. Phys. B544, 503 (1999).

[5] See, for example, Y. Nir and N. Seiberg, Phys. Lett. B 309, 337 (1993); M. Leurer, Y. Nir, and N. Seiberg, Nucl. Phys. B420, 468 (1994); L.E. Ibañez and G.G. Ross, Phys. Lett. B 332, 100 (1994); P. Binetruy and P. Ramond, ibid. 350, 49 (1995); V. Jain and R. Shrock, ibid. 352, 83 (1995); Stony Brook Report No. ITP-SB-95-22, hep-ph/9507238; Y. Nir, Phys. Lett. B 354, 107 (1995); E. Dudas, S. Pokorski, and C.A. Savoy, ibid. 356, 45 (1995); M.M. Robinson and J. Ziabicki, Phys. Rev. D 53, 5924 (1996); E. Dudas, S. Pokorski, and C.A. Savoy, Phys. Lett. B 369, 255 (1996); A. Pomarol and D. Tommasini, Nucl. Phys. B466, 3 (1996).

[6] See, for example: D.B. Kaplan and M. Schmaltz, Phys. Rev. D 49, 3741 (1994); M. Dine, R. Leigh, and A. Kagan, ibid. 48, 4269 (1993); L.J. Hall and H. Murayama, Phys. Rev. Lett. 75, 3985 (1995); C.D. Carone, L.J. Hall, and H. Murayama, Phys. Rev. D 53, 6282 (1996); P.H. Frampton and O.C.W. Kong, Phys. Rev. Lett. 77, 1699 (1996); P.H. Frampton and A. Rašin, hep-ph/9910522.

[7] See, for example, H. Arason, D.J. Castaño, E.J. Piard, and P. Ramond, Phys. Rev. D 47, 232 (1993).

[8] R. Barbieri, G. Dvali, and L.J. Hall, Phys. Lett. B 377, 76 (1996).

[9] R. Barbieri, L.J. Hall, and A. Romanino, Phys. Lett. B 401, 47 (1997).

[10] R. Barbieri, L.J. Hall, S. Raby, and A. Romanino, Nucl. Phys. B493, 3 (1997).

[11] H. Georgi and C. Jarlskog, Phys. Lett. 86B, 297 (1979).

[12] A. Aranda, C.D. Carone, and R.F. Lebed, Phys. Lett. B 474, 170 (2000).

[13] S. Coleman, Nucl. Phys. B310, 643 (1988); S. Giddings and A.
Strominger, ibid. B307, 854 (1988); G. Gilbert, ibid. B328, 159 (1989).

[14] M. Green and J. Schwarz, Phys. Lett. 149B, 117 (1984).

[15] R. Barbieri, P. Creminelli, and A. Romanino, Nucl. Phys. B559, 17 (1999).

[16] R. Dermisek and S. Raby, Phys. Rev. D 62, 015007 (2000).

[17] T. Banks, Nucl. Phys. B323, 90 (1989).

[18] L.M. Krauss and F. Wilczek, Phys. Rev. Lett. 62, 1221 (1989).

[19] J. Preskill, in Architecture of Fundamental Interactions at Short Distances, Proceedings of the Les Houches Summer School of Theoretical Physics, Session XLIV, 1985, edited by P. Ramond and R. Stora (Elsevier, New York, 1987), p. 235.

[20] L.E. Ibáñez and G.G. Ross, Phys. Lett. B 260, 291 (1991).

[21] T. Banks and M. Dine, Phys. Rev. D 45, 1424 (1992); J. Preskill, S.P. Trivedi, F. Wilczek, and M.B. Wise, Nucl. Phys. B363, 207 (1991).

[22] C.D. Carone and R.F. Lebed, Phys. Rev. D 60, 096002 (1999).

[23] A.D. Thomas and G.V. Wood, Group Tables (Shiva Publishing, Orpington, UK, 1980).

[24] R. Barbieri, L.J. Hall, D. Smith, A. Strumia, and N. Weiner, J. High Energy Phys. 12, 017 (1998).

[25] Super-Kamiokande Collaboration, Y. Fukuda et al., Phys. Rev. Lett. 81, 1562 (1998).

[26] J.N. Bahcall, P.I. Krastev, and A.Y. Smirnov, Phys. Rev. D 58, 096016 (1998).

[27] V. Barger, M.S. Berger, and P. Ohmann, Phys. Rev. D 47, 1093 (1993).

[28] Particle Data Group, C. Caso et al., Eur. Phys. J. C 3, 1 (1998).

[29] I. Antoniadis, C. Kounnas, and R. Lacaze, Nucl. Phys. B211, 216 (1983).

[30] W.H. Press, S.A. Teukolsky, W.T. Vetterling, and B.P. Flannery, Numerical Recipes in Fortran 77, 2nd ed. (Cambridge University Press, New York, 1992).

[31] K.S. Babu, C.N. Leung, and J. Pantaleone, Phys. Lett. B 319, 191 (1993). 
[32] F. Gabbiani, E. Gabrielli, A. Masiero, and L. Silvestrini, Nucl. Phys. B477, 321 (1996).

[33] A.G. Cohen, D.B. Kaplan, and A.E. Nelson, Phys. Lett. B 388, 588 (1996).
[34] C.D. Carone and L.J. Hall, Phys. Rev. D 56, 4198 (1997).

[35] L.J. Hall and N. Weiner, Phys. Rev. D 60, 033005 (1999).

[36] LSND Collaboration, C. Athanassopoulos et al., Phys. Rev. Lett. 81, 1774 (1998). 